\title{
The Nonlinear Meissner Effect in Unconventional Superconductors
}

\author{
D. Xu, S. K. Yip and J.A. Sauls \\ Department of Physics \& Astronomy, Northwestern University, Evanston, IL 60208 USA
}

(draft: July 17, 1994; revised: February 12, 1995)

\begin{abstract}
We examine the long-wavelength current response in anisotropic superconductors and show how the field-dependence of the Meissner penetration length can be used to detect the structure of the order parameter. Nodes in the excitation gap lead to a nonlinear current-velocity constitutive equation at low temperatures which is distinct for each symmetry class of the order parameter. The effective Meissner penetration length is linear in $H$ and exhibits a characteristic anisotropy for fields in the $a b$-plane that is determined by the positions of the nodes in momentum space. The nonlinear current-velocity relation also leads to an intrinsic magnetic torque for in-plane fields that are not parallel to a nodal or antinodal direction. The torque scales as $H^{3}$ for $T \rightarrow 0$ and has a characteristic angular dependence. We analyze the effects of thermal excitations, impurity scattering and geometry on the current response of a $d_{x^{2}-y^{2}}$ superconductor, and discuss our results in light of recent measurements of the low-temperature penetration length and in-plane magnetization of single-crystals of $\mathrm{YBa}_{2} \mathrm{Cu}_{3} \mathrm{O}_{7-\delta}$ and $\mathrm{LuBa}_{2} \mathrm{Cu}_{3} \mathrm{O}_{7-\delta}$.
\end{abstract}

\section{INTRODUCTION}

Recent measurements of the Meissner penetration depth [1] and Josephson interference effects in $\mathrm{YBa}_{2} \mathrm{Cu}_{3} \mathrm{O}_{7-\delta}$ [2] have been interpreted in support of a spin-singlet order parameter belonging to the one-dimensional, $d_{x^{2}-y^{2}}$ representation, $\Delta\left(\vec{p}_{f}\right)=\Delta_{0}\left(\hat{p}_{x}^{2}-\hat{p}_{u}^{2}\right)$, which breaks reflection symmetry in the basal plane. Such a pairing state has been proposed by several authors 3 河 based on arguments that the $\mathrm{CuO}$ materials are Fermi liquids close to an SDW instability.

If the cuprates have an order parameter that is unconventional, i.e. one that breaks additional symmetries of the normal state besides gauge symmetry, then the superconducting state is expected to exhibit a number of novel properties, including (i) gapless excitations below $T_{c}$, (ii) anomalous Josephson effects, (iii) exotic vortex structures and associated excitations, (iv) new collective modes, (v) sensitivity of superconducting coherence effects to defect scattering and (vi) multiple superconducting phases. [6.7] Many of these signatures of unconventional pairing have been observed in superfluid ${ }^{3} \mathrm{He}$, and in heavy fermion superconductors, notably $\mathrm{UPt}_{3}$. [8] The case for an unconventional order parameter in the cuprates, and particularly a $d_{x^{2}-y^{2}}$ state, is not settled; there are conflicting interpretations of closely related experiments, 22,9,10] variation in results that are presumably related to material quality or preparation, [11,12 and experimental results that are not easily accounted for within the $d_{x^{2}-y^{2}}$ model. [13,14]

In this paper we examine the long-wavelength current response in superconductors with an unconventional order parameter, and show how the field-dependence of the Meissner penetration length can be used to detect the structure of the order parameter. This report extends our earlier work on nonlinear supercurrents, [15, 16] and provides the relevant analysis that could not be included in our short reports. Specifically, we show (i) how the nodes in the excitation gap, whose multiplicity and position in momentum space depend on the symmetry class of the order parameter, lead to a nonlinear current-velocity constitutive equation at low temperatures $\left(T \ll T_{c}\right)$ which is unique and qualitatively distinct for each symmetry class. The effective Meissner penetration length is linear in $H$ and exhibits a characteristic anisotropy for fields in the ab-plane. (ii) This anisotropy is determined by the positions of the nodes in momentum space. For example, in the case of a $d_{x^{2}-y^{2}}$ state in a tetragonal material the anisotropy is precisely $1 / \sqrt{2}$, independent of the detailed shape of the Fermi surface or the gap. (iii) The nonlinear current-velocity relation leads to an intrinsic magnetic torque for in-plane fields that are not parallel to a nodal or antinodal direction. The torque scales as $H^{3}$ for $T \ll T_{c}$ and has a characteristic angular variation with a period of $\pi / 2$ (for tetragonal symmetry). The magnitude and angular dependence of this torque are calculated for thick superconducting films or slabs. (iv) We discuss the effects of thermal excitations, impurity scattering and geometry for observing these features in a $d_{x^{2}-y^{2}}$ superconductor. Recent measurements of the low-temperature, zero-field penetration length [1] are used to determine the relevant material parameters for $\mathrm{YBa}_{2} \mathrm{Cu}_{3} \mathrm{O}_{7-\delta}$, which are then used to estimate the magnitudes of the field-dependence of the penetration depth and the torque anisotropy at low temperatures. 
Our starting point is Fermi-liquid theory applied to anisotropic superconductors; section II includes the relevant theoretical framework needed to calculate the current response in unconventional superconductors. We derive formulas relating the equilibrium supercurrent to the magnetic field and discuss the linear response limit in section III. The nonlinear current-velocity constitutive equation is examined in section IV. A clean superconductor with a line of nodes in the gap has an anomalous contribution to the current which is a nonanalytic function of the condensate velocity, $\vec{v}_{s}$, at $T=0$. The relation of the anomalous current to the quasiparticle spectrum is discussed, and the contribution of this current to the Meissner penetration depth is obtained from solutions to the nonlinear London equation. The effects of impurity scattering and thermally excited quasiparticles on the anisotropy and field-dependence of the supercurrent are examined in detail; the signatures of the anomalous current survive thermal excitations and impurity scattering at sufficiently low temperatures and weak (or dilute) impurity scattering. We discuss our results in light of recent experiments on the low-temperature penetration depth [1] in single crystals of $\mathrm{YBa}_{2} \mathrm{Cu}_{3} \mathrm{O}_{6.95}$. An important conclusion is that if the linear temperature dependence of the penetration depth reported for $\mathrm{YBa}_{2} \mathrm{Cu}_{3} \mathrm{O}_{6.95}$ is due to the nodes of a $d_{x^{2}-y^{2}}$ order parameter, then the nonlinear Meissner effect, including the intrinsic anisotropy and field-dependence, should be observable for $T<1 \mathrm{~K}$ with a change in $\lambda_{a b}$ of approximately $30 \AA$ over the field range $0<H<H_{c 1} \simeq 200 \mathrm{G}$. In section $\mathrm{V}$ we discuss the nonlinear current, and associated in-plane magnetic torque, that develops for surface fields that are not aligned along a nodal or antinodal direction. The torque anisotropy (or transverse magnetization) is obtained from solutions to the nonlinear London equation at low temperatures. We also comment on a recent experimental report of a measurement of the in-plane magnetization [17] of a single crystal of $\mathrm{LuBa}_{2} \mathrm{Cu}_{3} \mathrm{O}_{7-\delta}$. In the rest of the introduction we briefly discuss the symmetry classes and unconventional order parameters for superconductors with tetragonal symmetry appropriate to the $\mathrm{CuO}$ superconductors (see Refs.( [18, 17, 19]) for detailed discussions.)

\section{Symmetries of the pairing state}

BCS superconductivity is based on a macroscopically occupied equal-time pairing amplitude $f_{\alpha \beta}\left(\vec{p}_{f}\right) \sim$ $\left\langle a_{\vec{p}_{f} \alpha} a_{-\vec{p}_{f} \beta}\right\rangle$, for quasiparticle pairs near the Fermi surface with zero total momentum and spin projections $\alpha$ and $\beta$. Fermi statistics requires that the order parameter obey the anti-symmetry condition, $f_{\alpha \beta}\left(\vec{p}_{f}\right)=-f_{\beta \alpha}\left(-\vec{p}_{f}\right)$, while inversion symmetry (if present) implies that the pairing amplitude decomposes into even-parity (spin-singlet) and odd-parity (spin-triplet) sectors. Furthermore, the pairing interaction separates into a sum over invariant bilinear products of basis functions for each irreducible representation of the point group. The resulting ground-state order parameter, barring the exceptional case of near degeneracy in two different channels, belongs to a single irreducible representation. For tetragonal symmetry there are four one-dimensional (1D) representations and one two-dimensional (2D) representation, and each of them occurs in both even- and odd-parity representations. The residual symmetry of the order parameter is just that of the basis functions for the $1 \mathrm{D}$ representations, but for the $2 \mathrm{D}$ representation there are three possible ground states with different residual symmetry groups. There is no evidence that we are aware of to support a spin-triplet order parameter in the $C u O$ superconductors; in fact the temperature dependence of the Knight shift in the cuprates 20,21] is argued to strongly favor a spin-singlet order parameter. [22 Thus, we limit the discussion to even-parity, spin-singlet states; however, most of the analysis and many of the main results for the current response are also valid for odd-parity states.

TABLE I. Even Parity Basis functions and Symmetry Classes for $D_{4 h}$

\begin{tabular}{clll}
\hline \hline Symmetry Class & Order Parameter: $\Delta\left(\vec{p}_{f}\right)$ & Residual Symmetry & Nodes \\
\hline$A_{1 g}$ & 1 & $D_{4 h} \times T$ & none \\
$A_{2 g}$ & $\hat{p}_{x} \hat{p}_{y}\left(\hat{p}_{x}^{2}-\hat{p}_{y}^{2}\right)$ & $D_{4}\left[C_{4}\right] \times C_{i} \times T$ & 8 lines: $\left|\hat{p}_{x}\right|= \pm\left|\hat{p}_{y}\right|, \hat{p}_{x}=0, \hat{p}_{y}=0$ \\
$B_{1 g}$ & $\hat{p}_{x}^{2}-\hat{p}_{y}^{2}$ & $D_{4}\left[D_{2}\right] \times C_{i} \times T$ & 4 lines: $\left|\hat{p}_{x}\right|= \pm\left|\hat{p}_{y}\right|$ \\
$B_{2 g}$ & $\hat{p}_{x} \hat{p}_{y}$ & $D_{4}\left[D_{2}^{\prime}\right] \times C_{i} \times T$ & 4 lines: $\hat{p}_{x}=0, \hat{p}_{y}=0$ \\
$E_{g}(1,0)$ & $\hat{p}_{z} \hat{p}_{x}$ & $D_{2}\left[C_{2}^{\prime}\right] \times C_{i} \times T$ & 3 lines: $\hat{p}_{z}=0, \hat{p}_{x}=0$ \\
$E_{g}(1,1)$ & $\hat{p}_{z}\left(\hat{p}_{x}+\hat{p}_{y}\right)$ & $D_{2}\left[C_{2}^{\prime \prime}\right] \times C_{i} \times T$ & 3 lines: $\hat{p}_{z}=0, \hat{p}_{x}+\hat{p}_{y}=0$ \\
$E_{g}(1, i)$ & $\hat{p}_{z}\left(\hat{p}_{x}+i \hat{p}_{y}\right)$ & $D_{4}[E] \times C_{i}$ & 1 line: $\hat{p}_{z}=0$ \\
\hline \hline
\end{tabular}

\footnotetext{
${ }^{1}$ The principal results and conclusions presented here are not qualitatively modified by a-b anisotropy; the quanitative effects of $\mathrm{a}-\mathrm{b}$ anisotropy will be discussed elsewhere.
} 
Table I summarizes the symmetry classes of the order parameter for spin-singlet pairing. All of the 1D representations have residual symmetry groups which include four-fold rotations combined with appropriate elements of the gauge groups. The states $E_{g}(1,0)$ and $E_{g}(1,1)$ have a residual symmetry group that allows only two-fold rotations. The resulting supercurrent, or superfluid density tensor, for such states is in general strongly anisotropic in the basal plane. The $2 \mathrm{D}$ order parameter, $E_{g}(1, i)$, preserves the four-fold rotational symmetry, but breaks time-reversal symmetry.

Although the $B_{1 g}\left(d_{x^{2}-y^{2}}\right)$ and $B_{2 g}\left(d_{x y}\right)$ order parameters break the $C_{4}$ rotational symmetry of the $\mathrm{CuO}$ planes, a combined $C_{4}$ rotation and gauge transformation by $e^{i \pi}$ is a symmetry. Since many properties of the superconducting state depend only on Fermi-surface averages of $\left|\Delta\left(\vec{p}_{f}\right)\right|^{2}$, the broken rotational symmetry is not easy to observe. In particular, the London penetration depth tensor is cylindrically symmetric for any of the 1D pairing states listed in Table I. Furthermore, all of the unconventional gaps in Table I yield a linear temperature dependence at $T \ll T_{c}$ for the zero-field penetration depth (in the clean limit).

A distinguishing feature of each phase, which is a consequence of their particular broken symmetries, is that the nodes of each gap are located in different positions in $\vec{p}$-space. A point that we make below is that the field-dependence of the supercurrent may be used to locate the positions of the nodal lines (or points) of an unconventional gap in momentum space. This gap spectroscopy is possible at low temperatures, $T \ll T_{c}$, and is based on features which are intrinsic to nearly all unconventional BCS states in tetragonal or orthorhombic structures.

\section{FERMI-LIQUID THEORY OF SUPERCONDUCTIVITY}

Our starting point for calculations of the current response is the Fermi-liquid theory of superconductivity. This theory is general enough to include real materials effects of Fermi-surface anisotropy, impurity scattering and inelastic scattering from phonons and quasiparticles, in addition to unconventional pairing. A basic feature of the Fermi-liquid theory of superconductivity (c.f. Refs.( [23 25]) for a more detailed discussion of the formulation of Fermi-liquid theory.) is that for low excitation energies $\left(\hbar \omega, k_{B} T, \hbar q v_{f}, \Delta\right) \ll E_{f}$, the wave nature of the quasiparticle excitations is unimportant and can be eliminated by integrating the full Matsubara Green's function over the quasiparticle momentum (or kinetic energy) in the low-energy band around the Fermi surface,

$$
g_{\alpha \beta}\left(\vec{p}_{f}, \vec{R} ; \epsilon_{n}\right)=-\int_{-\omega_{c}}^{\omega_{c}} d \xi_{\vec{p}} \int_{0}^{\beta} d \tau e^{i \epsilon_{n} \tau / \hbar} \int d \vec{r} e^{-i \vec{p} \cdot \vec{r} / \hbar}<T_{\tau} \psi_{\alpha}(\vec{R}+\vec{r} / 2, \tau) \psi_{\beta}^{\dagger}(\vec{R}-\vec{r} / 2,0)>,
$$

where $\xi_{\vec{p}}=v_{f}\left(\vec{p}_{f}\right)\left(|\vec{p}|-\left|\vec{p}_{f}\right|\right)$ is the normal-state quasiparticle excitation energy for momentum $\vec{p}$ nearest to the position $\vec{p}_{f}$ on the Fermi surface and $\vec{v}_{f}\left(\vec{p}_{f}\right)$ is the quasiparticle velocity at the point $\vec{p}_{f}$. The resulting quasiclassical propogator is a function of the momentum direction $\vec{p}_{f}$ on the Fermi surface, the center of mass coordinate $\vec{R}$ and the Matsubara energy $\epsilon_{n}=(2 n+1) \pi T$. The pairing correlations are described by the $\xi$-integrated anomalous Green's functions,

$$
f_{\alpha \beta}\left(\vec{p}_{f}, \vec{R} ; \epsilon_{n}\right)=-\int_{-\omega_{c}}^{\omega_{c}} d \xi_{\vec{p}} \int_{0}^{\beta} d \tau e^{i \epsilon_{n} \tau / \hbar} \int d \vec{r} e^{-i \vec{p} \cdot \vec{r} / \hbar}<T_{\tau} \psi_{\alpha}(\vec{R}+\vec{r} / 2, \tau) \psi_{\beta}(\vec{R}-\vec{r} / 2,0)>.
$$

The low-energy quasiparticle spectrum, combined with charge conservation and gauge invariance, allows one to formulate observables in terms of the quasiclassical Green's function and material parameters defined on the Fermi surface. For example, the equilibrium current is given by

$$
\vec{j}_{s}(\vec{R})=-e N_{f} \int d \vec{p}_{f} \vec{v}_{f}\left(\vec{p}_{f}\right) T \sum_{n} \frac{1}{2} \operatorname{Tr}\left\{\hat{\tau}_{3} \hat{g}\left(\vec{p}_{f}, \vec{R} ; \epsilon_{n}\right)\right\},
$$

where $N_{f}$ is the single-spin density of states at the Fermi level and the integration is over the Fermi surface with a weight factor of the angle-resolved density of states normalized to unity. We have introduced the $4 \times 4$ quasiclassical functions in 'spin $\times$ particle-hole' space; a convenient representation for the particle-hole and spin structure of the propagator is,

$$
\hat{g}=\left(\begin{array}{cc}
g\left(\vec{p}_{f}, \vec{R} ; \epsilon_{n}\right)+\vec{g}\left(\vec{p}_{f}, \vec{R} ; \epsilon_{n}\right) \cdot \vec{\sigma} & f\left(\vec{p}_{f}, \vec{R} ; \epsilon_{n}\right) i \sigma_{y}+\vec{f}\left(\vec{p}_{f}, \vec{R} ; \epsilon_{n}\right) \cdot i \vec{\sigma} \sigma_{y} \\
f\left(-\vec{p}_{f}, \vec{R} ; \epsilon_{n}\right)^{*} i \sigma_{y}-\vec{f}\left(-\vec{p}_{f}, \vec{R} ; \epsilon_{n}\right)^{*} \cdot i \sigma_{y} \vec{\sigma} & g\left(-\vec{p}_{f}, \vec{R} ;-\epsilon_{n}\right)-\vec{g}\left(-\overrightarrow{p_{f}}, \vec{R} ;-\epsilon_{n}\right) \cdot \sigma_{y} \vec{\sigma} \sigma_{y}
\end{array}\right) .
$$

This matrix structure represents the remaining quantum mechanical degrees of freedom; the coherence of particle and hole states is contained in the off-diagonal elements in eq.(11). The diagonal components are separated into spinscalar, $g$, and spin-vector, $\vec{g}$, components. The scalar component determines the current response, while the vector 
components determine the spin-paramagnetic response. The off-diagonal propagator separates into spin-singlet, $f$, and spin-triplet, $\vec{f}$, pairing amplitudes, which are coupled to the diagonal propagators through the quasiclassical transport equation,

$$
\hat{Q}[\hat{g}, \hat{\sigma}] \equiv\left[i \epsilon_{n} \hat{\tau}_{3}-\hat{\sigma}\left(\vec{p}_{f}, \vec{R} ; \epsilon_{n}\right), \hat{g}\left(\vec{p}_{f}, \vec{R} ; \epsilon_{n}\right)\right]+i \vec{v}_{f} \cdot \vec{\nabla} \hat{g}\left(\vec{p}_{f}, \vec{R} ; \epsilon_{n}\right)=0,
$$

first derived by Eilenberger [26] by eliminating the high-energy, short-distance structure of the full Green's function in Gorkov's equations. 27] The transport equation is supplemented by the normalization condition,

$$
\hat{g}\left(\vec{p}_{f}, \vec{R} ; \epsilon_{n}\right)^{2}=-\pi^{2} \hat{1}
$$

which eliminates many unphysical solutions from the general set of solutions to the transport equation. [26]

The self energy, $\hat{\sigma}$, has an expansion (Fig. 1) in terms of $\hat{g}$ (solid lines) and renormalized vertices describing the interactions between quasiparticles, phonons (wiggly lines), impurities and external fields. An essential feature of Fermi liquid theory is that this expansion is based on a set of small expansion parameters, small $\sim k_{B} T_{c} / E_{f}, \hbar / p_{f} \xi_{0}, \ldots \ll 1$, which are the relevant low-energy (e.g. pairing energy) or long-wavelength (e.g. coherence length) scales compared to the characteristic high-energy (e.g. Fermi energy) or short-wavelength (e.g. Fermi wavelength) scales. [23] The leading order contributions to the self energy are represented in Fig.1. Diagram (1a) is the zeroth-order in small and represents the band-structure potential of the quasiparticles. This term is included as Fermi-surface data for $\vec{p}_{f}, \vec{v}_{f}$ and $N_{f}$, which is taken from experiment or defined by a model for the band-structure. Diagram (1b) is first-order in small and represents Landau's Fermi-liquid interactions (diagonal in particle-hole space), and the electronic pairing interactions (off-diagonal in particle-hole space), or mean-field pairing self-energy (also the 'order parameter' or 'gap function'). Diagram (1b') represents the leading-order phonon contribution to the electronic self energy (diagonal) and pairing self-energy (off-diagonal); however, we confine our discussion to electronically driven superconductivity with a frequency-independent interaction.
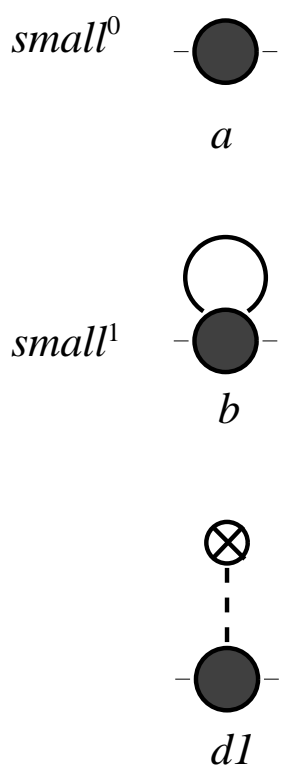
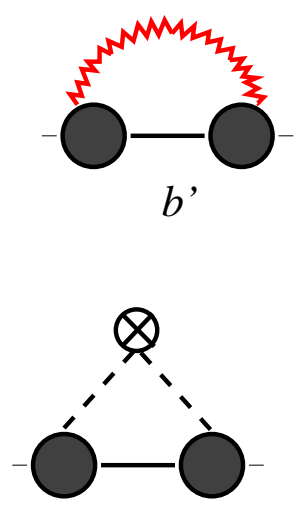

$d 2$
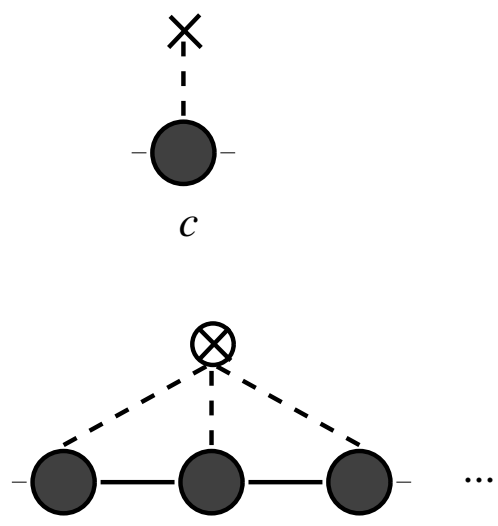

d3

Fig. 1 Leading order contributions to the quasiclassical self-energy.

In the spin-singlet channel, the order parameter satisfies the gap equation,

$$
\Delta\left(\vec{p}_{f}, \vec{R}\right)=\int d \vec{p}_{f}^{\prime} V\left(\vec{p}_{f}, \vec{p}_{f}^{\prime}\right) T \sum_{\epsilon_{n}} f\left(\vec{p}_{f}^{\prime}, \vec{R} ; \epsilon_{n}\right),
$$

where $f\left(\vec{p}_{f}, \vec{R} ; \epsilon_{n}\right)$ is the spin-singlet pairing amplitude and $V\left(\vec{p}_{f}, \vec{p}_{f}^{\prime}\right)$ represents the electronic pairing interaction; this function may be expanded in basis functions for the irreducible representations of the point group,

$$
V\left(\vec{p}_{f}, \vec{p}_{f}^{\prime}\right)=\sum_{\alpha}^{\text {irrep }} V_{\alpha} \sum_{i=1}^{d_{\alpha}} \mathcal{Y}_{\alpha i}\left(\vec{p}_{f}\right) \mathcal{Y}_{\alpha i}\left(\vec{p}_{f}^{\prime}\right),
$$


where the parameter, $V_{\alpha}$, is the pairing interaction in the channel labeled by the $\alpha$ th irreducible representation, and the corresponding basis functions, $\left\{\mathcal{Y}_{\alpha i}\left(\vec{p}_{f}\right) \mid i=1, \ldots, d_{\alpha}\right\}$, are orthonormal, $\left\langle\mathcal{Y}_{\alpha i}\left(\vec{p}_{f}\right) \mathcal{Y}_{\beta j}^{*}\left(\vec{p}_{f}\right)\right\rangle_{\vec{p}_{f}}=\delta_{\alpha \beta} \delta_{i j}$, where the Fermi surface average is defined by $\left\langle A\left(\vec{p}_{f}\right)>_{\vec{p}_{f}}=\int d \vec{p}_{f} A\left(\vec{p}_{f}\right)\right.$.

\section{Impurity scattering}

The summation of diagrams (1d) gives the leading-order self energy from a random distribution of impurities in terms of the impurity t-matrix, 28, 29.

$$
\begin{gathered}
\hat{\sigma}_{i m p}\left(\vec{p}_{f} ; \epsilon_{n}\right)=n_{i m p} \hat{t}\left(\vec{p}_{f}, \vec{p}_{f} ; \epsilon_{n}\right) \\
\hat{t}\left(\vec{p}_{f}, \vec{p}_{f}^{\prime} ; \epsilon_{n}\right)=\hat{u}\left(\vec{p}_{f}, \vec{p}_{f}^{\prime}\right)+N_{f} \int d \vec{p}_{f}^{\prime \prime} \hat{u}\left(\vec{p}_{f}, \vec{p}_{f}^{\prime \prime}\right) \hat{g}\left(\vec{p}_{f}^{\prime \prime} ; \epsilon_{n}\right) \hat{t}\left(\vec{p}_{f}^{\prime \prime}, \vec{p}_{f}^{\prime} ; \epsilon_{n}\right) .
\end{gathered}
$$

The first term is the matrix element of the impurity potential between quasiparticles at points $\vec{p}_{f}$ and $\vec{p}_{f}^{\prime}$ on the Fermi surface, $n_{i m p}$ is the impurity concentration, and the intermediate states are defined by the self-consistently determined quasiclassical propagator.

For a spin-singlet superconductor with non-magnetic impurities, $\hat{u}\left(\vec{p}_{f}, \vec{p}_{f}^{\prime}\right)=u\left(\vec{p}_{f}, \vec{p}_{f}^{\prime}\right) \hat{1}$, and the terms in $\hat{\sigma}_{i m p}$ that contribute in the transport equation lead to a renormalization of the Matsubara frequency and gap function; $i \tilde{\epsilon}_{n}=i \epsilon_{n}-\sigma_{i m p}\left(\vec{p}_{f} ; \epsilon_{n}\right)$ and $\tilde{\Delta}\left(\vec{p}_{f} ; \epsilon_{n}\right)=\Delta\left(\vec{p}_{f}\right)+\Delta_{i m p}\left(\vec{p}_{f} ; \epsilon_{n}\right)$. Thus, the solution to the transport equation and normalization condition for the propagator becomes,

$$
\hat{g}\left(\vec{p}_{f} ; \epsilon_{n}\right)=-\pi \frac{i \tilde{\epsilon}_{n}\left(\vec{p}_{f} ; \epsilon_{n}\right) \hat{\tau}_{3}-\hat{\tilde{\Delta}}\left(\vec{p}_{f} ; \epsilon_{n}\right)}{\sqrt{\tilde{\epsilon}_{n}\left(\vec{p}_{f} ; \epsilon_{n}\right)^{2}+\left|\tilde{\Delta}\left(\vec{p}_{f} ; \epsilon_{n}\right)\right|^{2}}} .
$$

In the second-order Born approximation for the impurity t-matrix (this is not essential, but simplifies the following discussion). The impurity renormalization of the off-diagonal self-energy is given by,

$$
\tilde{\Delta}\left(\vec{p}_{f} ; \epsilon_{n}\right)=\Delta\left(\vec{p}_{f}\right)+\int d \vec{p}_{f}^{\prime} w\left(\vec{p}_{f}, \vec{p}_{f}^{\prime}\right) \frac{\tilde{\Delta}\left(\vec{p}_{f}^{\prime} ; \epsilon_{n}\right)}{\sqrt{\tilde{\epsilon}_{n}^{2}+\left|\tilde{\Delta}\left(\vec{p}_{f} ; \epsilon_{n}\right)\right|^{2}}},
$$

where $w\left(\vec{p}_{f}, \vec{p}_{f}^{\prime}\right)=2 \pi n_{i m p} N_{f}\left|u\left(\vec{p}_{f}, \vec{p}_{f}^{\prime}\right)\right|^{2}$ is the scattering rate in the Born approximation. Note that the integral equation for $\tilde{\Delta}\left(\vec{p}_{f} ; \epsilon_{n}\right)$ has the mean-field order parameter, $\Delta\left(\vec{p}_{f}\right)$, as the driving term. The scattering rate $w\left(\vec{p}_{f}, \vec{p}_{f}^{\prime}\right)$ has the full symmetry of the normal metal; thus, it too can be expanded in basis functions for the irreducible representations of the point group,

$$
w\left(\vec{p}_{f}, \vec{p}_{f}^{\prime}\right)=\sum_{\alpha}^{i r r e p} \frac{1}{2 \tau_{\alpha}} \sum_{i=1}^{d_{\alpha}} \mathcal{Y}_{\alpha i}\left(\vec{p}_{f}\right) \mathcal{Y}_{\alpha i}^{*}\left(\vec{p}_{f}^{\prime}\right),
$$

where $1 / 2 \tau_{\alpha}$ is the scattering rate for channel $\alpha$. The integral equation for the renormalized order parameter separates into algebraic equations for each representation,

$$
\tilde{\Delta}_{\alpha i}=\int d \vec{p}_{f} \mathcal{Y}_{\alpha i}^{*}\left(\vec{p}_{f}\right) \Delta\left(\vec{p}_{f}\right)+\frac{1}{2 \tau_{\alpha}} \int d \vec{p}_{f} \frac{\mathcal{Y}_{\alpha i}^{*}\left(\vec{p}_{f}\right) \tilde{\Delta}\left(\vec{p}_{f} ; \epsilon_{n}\right)}{\sqrt{\tilde{\epsilon}_{n}^{2}+\left|\tilde{\Delta}\left(\vec{p}_{f} ; \epsilon_{n}\right)\right|^{2}}} .
$$

The driving term is non-zero only for the irreducible representation corresponding to $\Delta\left(\vec{p}_{f}\right)$. Thus, the resulting solution for the impurity renormalized order parameter necessarily has the same orbital symmetry as the mean-field order parameter; and the magnitude of the impurity renormalization is determined by the scattering probability for scattering in the same channel as that of $\Delta\left(\vec{p}_{f}\right)$. [30] The argument also holds for the full t-matrix.

For isotropic ('s-wave') impurity scattering the renormalized Matsubara frequency and order parameter become

$$
\tilde{\epsilon}_{n}=\epsilon_{n}+\frac{1}{2 \tau}\left\langle\frac{\tilde{\epsilon}_{n}}{\sqrt{\tilde{\epsilon}_{n}^{2}+\left|\tilde{\Delta}\left(\vec{p} f ; \epsilon_{n}\right)\right|^{2}}}\right\rangle,
$$




$$
\tilde{\Delta}\left(\vec{p}_{f} ; \epsilon_{n}\right)=\Delta\left(\vec{p}_{f}\right)+\frac{1}{2 \tau}\left\langle\frac{\tilde{\Delta}\left(\vec{p}_{f}^{\prime} ; \epsilon_{n}\right)}{\sqrt{\tilde{\epsilon}_{n}^{2}+\left|\tilde{\Delta}\left(\vec{p}_{f}^{\prime} ; \epsilon_{n}\right)\right|^{2}}}\right\rangle_{\vec{p}_{f}} .
$$

Thus, for an s-wave order parameter these equations give identical renormalization factors for both the Matsubara frequency and the order parameter, i.e.

$$
\frac{\tilde{\epsilon}_{n}}{\epsilon_{n}}=\frac{\tilde{\Delta}\left(\epsilon_{n}\right)}{\Delta}=Z\left(\epsilon_{n}\right)=1+\frac{1}{2 \pi \tau} \frac{1}{\sqrt{\epsilon_{n}^{2}+\Delta^{2}}} \quad(s-\text { wave })
$$

in which case the impurity renormalization drops out of the equilibrium propagator and gap equation. [31, 32] However, s-wave superconductors are exceptional; for any unconventional order parameter impurity scattering is pairbreaking. 29

Consider an unconventional superconductor with impurities in which the scattering is dominated by the identity representation. If there is an element of the point group, $\mathcal{R}$, which changes the sign of $\Delta\left(\vec{p}_{f}\right)$, i.e. $\Delta\left(\vec{p}_{f}\right) \stackrel{\mathcal{R}}{\longrightarrow}-\Delta\left(\vec{p}_{f}\right)$, then from eq.(16) the impurity renormalization of the order parameter vanishes identically: $\tilde{\Delta}\left(\vec{p}_{f} ; \epsilon_{n}\right)=\Delta\left(\vec{p}_{f}\right)$. The cancellation between the impurity renormalization factors for the Matsubara frequency and order parameter no longer occurs, with the consequence that impurity scattering suppresses both $T_{c}$ and the magnitude of the order parameter.

For isotropic impurity scattering (not restricted to the Born approximation), the renormalization factor for the Matsubara frequency, $\tilde{\epsilon}_{n} / \epsilon_{n}=Z\left(\epsilon_{n}\right)$, is independent of position on the Fermi surface and given by

$$
Z\left(\epsilon_{n}\right)=1+\Gamma_{u} \frac{Z\left(\epsilon_{n}\right) \mathcal{D}\left(\epsilon_{n}\right)}{\operatorname{ctn}^{2}\left(\delta_{0}\right)+\left(Z\left(\epsilon_{n}\right) \epsilon_{n} \mathcal{D}\left(\epsilon_{n}\right)\right)^{2}},
$$

with

$$
\mathcal{D}\left(\epsilon_{n}\right)=\left\langle\frac{1}{\sqrt{Z\left(\epsilon_{n}\right)^{2} \epsilon_{n}^{2}+\left|\Delta\left(\vec{p}_{f}\right)\right|^{2}}}\right\rangle_{\vec{p}_{f}}
$$

where $\Gamma_{u}=n_{i m p} / \pi N_{f}$ and $\delta_{0}=\tan ^{-1}\left(\pi N_{f} u_{0}\right)$ is the s-wave scattering phase shift in the normal state. In the Born limit, $\delta_{0} \rightarrow \pi N_{f} u_{0}$, while in the strong scattering limit $\left(N_{f} u_{0} \rightarrow \infty\right)$ we obtain the unitarity limit, $\delta_{0} \rightarrow \pi / 2$.

Given the gap function, $\Delta\left(\vec{p}_{f}\right)$, the impurity renormalization is easily calculated. The magnitude and temperature dependence of the order parameter are calculated self-consistently from the mean-field gap equation,

$$
\Delta\left(\vec{p}_{f}\right)=\int d \vec{p}_{f}^{\prime} V\left(\vec{p}_{f}, \vec{p}_{f}^{\prime}\right) \pi T \sum_{\epsilon_{n}}^{\left|\epsilon_{n}\right|<\omega_{c}} \frac{\Delta\left(\vec{p}_{f}^{\prime}\right)}{\sqrt{Z\left(\epsilon_{n}\right)^{2} \epsilon_{n}^{2}+\left|\Delta\left(\vec{p}_{f}^{\prime}\right)\right|^{2}}} .
$$

The linearized gap equation determines $T_{c}$ in terms of the pairing interaction, frequency cutoff $\omega_{c}$ and impurity scattering rate. At $T_{c}$ only the dominant pairing channel $\alpha$ is relevant and the linearized gap equation becomes,

$$
\frac{1}{V_{\alpha}}=\pi T_{c} \sum_{\epsilon_{n}}^{\left|\epsilon_{n}\right|<\omega_{c}} \frac{1}{\left|\epsilon_{n}\right|+\Gamma},
$$

where $\Gamma=\Gamma_{u} \sin ^{2} \delta_{0}$ is the pair-breaking parameter,

$$
\Gamma=\left\{\begin{array}{l}
\Gamma_{u} \sin ^{2} \delta_{0}=\frac{1}{2 \tau}=\pi n_{i m p} N_{f} u_{0}^{2} \quad, \quad \text { (Born limit) } \\
\Gamma_{u}=\frac{n_{i m p}}{\pi N_{f}}
\end{array} \quad, \quad\right. \text { (unitarity limit). }
$$

For $\Gamma=0$, this equation determines the clean-limit value of the transition temperature, $T_{c o}$. Eliminating the pairing interaction and cutoff gives the well-known Abrikosov-Gorkov formula, [33] except that the pairbreaking parameter is determined by non-magnetic scattering, [6]

$$
\psi\left(\frac{1}{2}+\frac{\Gamma}{2 \pi T_{c}}\right)-\psi\left(\frac{1}{2}\right)=\ln \left(\frac{T_{c o}}{T_{c}}\right)
$$

where $\psi(z)$ is the digamma function. 
Finally, the linearized gap equation is used to eliminate the pairing interaction and cutoff in favor of $T_{c}$ in the full gap equation (20). For pairing in a 1D representation, $V\left(\vec{p}_{f}, \vec{p}_{f}^{\prime}\right)=V e\left(\vec{p}_{f}\right) e\left(\vec{p}_{f}^{\prime}\right)^{*}$, the order parameter is $\Delta\left(\vec{p}_{f}\right)=\Delta e\left(\vec{p}_{f}\right)$, where $\Delta$ is obtained from eq.(20). Multiplying eq.(20) by $e\left(\vec{p}_{f}\right)^{*}$, integrating over the Fermi surface, and adding and subtracting the RHS of the linearized gap equation (with $T_{c} \rightarrow T$ ) to eliminate $V$ gives,

$$
\begin{aligned}
& {\left[\ln \left(T / T_{c}\right)+\psi\left(\frac{1}{2}+\frac{\Gamma}{2 \pi T}\right)-\psi\left(\frac{1}{2}+\frac{\Gamma}{2 \pi T_{c}}\right)\right]=} \\
& 2 \pi T \sum_{n=0}^{\infty}\left[\left\langle\frac{\left|e\left(\vec{p}_{f}\right)\right|^{2}}{\sqrt{Z\left(\epsilon_{n}\right)^{2} \epsilon_{n}^{2}+\Delta^{2}\left|e\left(\vec{p}_{f}\right)\right|^{2}}}\right\rangle_{\vec{p}_{f}}-\frac{1}{\epsilon_{n}+\Gamma}\right],
\end{aligned}
$$

which is solved self-consistently with eq.(18) to give $Z\left(\epsilon_{n}\right)$ and $\Delta$ as a function of $T / T_{c}$ and $\Gamma$.

\section{Gauge-invariant coupling to the condensate flow field}

The self-energy term representing the diamagnetic coupling of quasiparticles to a static magnetic field (Fig. 1c) is determined by local gauge invariance. Under a gauge transformation of the Fermion fields, $\psi(\vec{r}) \rightarrow \psi(\vec{r}) e^{-i \Lambda(\vec{r}) / 2}$, the quasiclassical propagator transforms as

$$
\hat{g} \stackrel{\Lambda}{\longrightarrow} \hat{g}^{\prime}=\hat{U}(\Lambda)^{\dagger} \hat{g} \hat{U}(\Lambda),
$$

where $\hat{U}(\Lambda)=\exp \left(+\frac{i}{2} \Lambda(\vec{R}) \hat{\tau}_{3}\right)$, as does the self-energy and order parameter. 23] Applying this transformation to the transport equation (5) gives $\hat{U}(\Lambda)^{\dagger} \hat{Q}[\hat{g}, \hat{\sigma}] \hat{U}(\Lambda)=\hat{Q}\left[\hat{g}^{\prime}, \hat{\sigma}^{\prime}+\hat{\sigma}_{\nabla}\right]$. Thus, the form of the transport equation is invariant, but the local gauge field generates an additional self-energy, $\hat{\sigma}_{\nabla}=-i \hat{U}(\Lambda)^{\dagger} \vec{v}_{f}\left(\vec{p}_{f}\right) \cdot \vec{\nabla} \hat{U}(\Lambda)$. This property of the transport equation is used to eliminate the phase degree of freedom of the order parameter in favor of a spatially varying flow field. We parametrize the spatial variations in terms of a physical gauge, the phase $\chi(\vec{R})$, and the local amplitude, $\Delta_{0}\left(\vec{p}_{f} ; \vec{R}\right)=|\Delta(\vec{R})| e\left(\vec{p}_{f}\right)$

$$
\hat{\Delta}\left(\vec{p}_{f}, \vec{R}\right)=\hat{U}[\chi(\vec{R})] \hat{\Delta}_{0}\left(\vec{p}_{f}, \vec{R}\right) \hat{U}^{\dagger}[\chi(\vec{R})] .
$$

Thus, the transport equation becomes

$$
\left[i \epsilon_{n} \hat{\tau}_{3}-\hat{\Delta}_{0}-\hat{\sigma}_{v}-\hat{\sigma}^{\prime}, \hat{g}^{\prime}\right]+i \vec{v}_{f}\left(\vec{p}_{f}\right) \cdot \vec{\nabla} \hat{g}^{\prime}=0
$$

where $\hat{\sigma}_{v}=\frac{1}{2} \vec{v}_{f}\left(\vec{p}_{f}\right) \cdot \vec{\nabla} \chi \hat{\tau}_{3}$. The diamagnetic coupling to a magnetic field, $\vec{b}=\vec{\nabla} \times \vec{A}$, is then determined by gauge invariance, and can be represented in terms of the gauge-invariant condensate flow field,

$$
\vec{v}_{s}=\frac{1}{2}\left(\vec{\nabla} \chi+\frac{2 e}{c} \vec{A}\right)
$$

and the self-energy,

$$
\hat{\sigma}_{v}=\vec{v}_{f}\left(\vec{p}_{f}\right) \cdot \vec{v}_{s}(\vec{R}) \hat{\tau}_{3} .
$$

In the Meissner geometry ( $\vec{H}$ parallel to the interface) the driving term associated with the applied surface field is of order,

$$
\left|\frac{\sigma_{v}}{\pi T_{c}}\right| \sim \frac{\frac{e}{c} v_{f} A}{\pi T_{c}} \sim \frac{H}{H_{c}}
$$

where $H_{c} \sim \phi_{0} /(\xi \lambda)$ is the thermodynamic critical field. This term should be compared to the gradient term arising from spatial variations of the screening current,

$$
\left|\frac{\vec{v}_{f} \cdot \vec{\nabla} g}{\pi T_{c}}\right| \sim\left|\frac{\vec{v}_{f} \cdot \vec{\nabla}\left(\sigma_{v} / \Delta\right)}{\pi T_{c}}\right| \sim \frac{\xi}{\lambda} \frac{H}{H_{c}} .
$$

In the strong type II limit the velocity field is effectively uniform on the scale of the coherence length so we are generally justified in dropping the gradient term in eq. 27). 


\section{Linear response}

The linear response limit is simply obtained from a perturbation expansion of the propagator, transport equation and normalization condition, and is expected to be valid for low magnetic fields, $\left|\sigma_{v} / \pi T_{c}\right| \sim H / H_{c} \ll 1$. Assume an expansion of the form, $\hat{g}=\hat{g}_{0}+\hat{g}_{1}+\ldots$, where $\hat{g}_{0}$ is the zero-field solution to the transport equation given by eq.(111), and $\hat{g}_{1}$ is the first-order correction to the propagator, formally of order $\left|\hat{g}_{1}\right| \sim \mathcal{O}\left|\left(\sigma_{v} / \Delta\right) \hat{g}_{0}\right|$. The linearized transport equation and normalization condition,

$$
\left[i \tilde{\epsilon}_{n} \hat{\tau}_{3}-\hat{\tilde{\Delta}}, \hat{g}_{1}\right]-\left[\hat{\sigma}_{v}, \hat{g}_{0}\right]=0 \quad, \quad\left\{\hat{g}_{0}, \hat{g}_{1}\right\}=0,
$$

are inverted with the aid of eqs. (11) and (6) to give

$$
\hat{g}_{1}=\pi \hat{\sigma}_{v} \frac{|\tilde{\Delta}|^{2}-i \tilde{\epsilon}_{n} \hat{\tau}_{3} \hat{\tilde{\Delta}}}{\left[\tilde{\epsilon}_{n}^{2}+\left|\tilde{\Delta}\left(\vec{p}_{f}, \epsilon_{n}\right)\right|^{2}\right]^{3 / 2}} .
$$

The resulting supercurrent calculated from eq. (3) can be written in terms of the superfluid density tensor,

$$
\left(\stackrel{\leftrightarrow}{\rho}_{s}\right)_{i j}=2 N_{f} \int d \vec{p}_{f} \Phi\left(\vec{p}_{f}\right) v_{f}^{i}\left(\vec{p}_{f}\right) v_{f}^{j}\left(\vec{p}_{f}\right)
$$

where

$$
\Phi\left(\vec{p}_{f}\right)=\pi T \sum_{\epsilon_{n}} \frac{\left|\tilde{\Delta}\left(\vec{p}_{f}, \epsilon_{n}\right)\right|^{2}}{\left[\tilde{\epsilon}_{n}^{2}+\left|\tilde{\Delta}\left(\vec{p}_{f}, \epsilon_{n}\right)\right|^{2}\right]^{3 / 2}},
$$

which reduces to the angle-dependent Yosida function in the clean limit.

\section{Fermi-liquid effects}

Fermi-liquid effects arise from the leading order electronic self-energy (diagram 1b). For the diamagnetic response the most important Fermi-liquid effect is the screening correction to the diamagnetic current; the relevant self energy is, $\hat{\sigma}_{b}\left(\vec{p}_{f}, \vec{R}\right)=\sigma_{b}\left(\vec{p}_{f}, \vec{R}\right) \hat{\tau}_{3}$, with

$$
\sigma_{b}\left(\vec{p}_{f}, \vec{R}\right)=\int d \vec{p}_{f}^{\prime} A^{c u r}\left(\vec{p}_{f}, \vec{p}_{f}^{\prime}\right) T \sum_{\epsilon_{n}} g\left(\vec{p}_{f}^{\prime}, \vec{R} ; \epsilon_{n}\right),
$$

where $A^{\text {cur }}\left(\vec{p}_{f}, \vec{p}_{f}^{\prime}\right)$ is the dimensionless quasiparticle interaction.

Fermi liquid effects can contribute substantial temperature-dependent corrections to the penetration depth as the gap opens and the number of thermal quasiparticles drops rapidly. We include formulas for the Fermi-liquid correction to the supercurrent for a model uniaxial Fermi surface. The position on the Fermi surface can be parametrized by the direction of the Fermi wavevector, $\hat{p}$, and the Fermi velocity is given by,

$$
\vec{v}_{f}=v_{f}^{\|}\left(\hat{p}_{x} \hat{x}+\hat{p}_{y} \hat{y}\right)+v_{f}^{\perp} \hat{p}_{z} \hat{z} .
$$

Similarly, the quasiparticle interaction is parametrized by two Landau parameters corresponding to current flow in the basal plane and along the $\hat{z}$-axis,

$$
A^{\text {cur }}\left(\vec{p}_{f}, \vec{p}_{f}^{\prime}\right)=A^{\|}\left(\hat{p}_{x} \hat{p}_{x}^{\prime}+\hat{p}_{y} \hat{p}_{y}^{\prime}\right)+A^{\perp}\left(\hat{p}_{z} \hat{p}_{z}^{\prime}\right) .
$$

The linear response result for the supercurrent is easily obtained from eq.(33) with the replacement $\hat{\sigma}_{v} \rightarrow \hat{\sigma}_{s c}=$ $\vec{v}_{f}\left(\vec{p}_{f}\right) \cdot \vec{v}_{s}+\sigma_{b}\left(\vec{p}_{f}\right)$. The resulting current is given by

$$
\vec{j}_{s}(\vec{R})=-2 e N_{f} \int d \vec{p}_{f} \vec{v}_{f}\left(\vec{p}_{f}\right) \Phi\left(\vec{p}_{f}\right) \sigma_{s c}\left(\vec{p}_{f}\right),
$$

with the screening field satisfying the self-consistency equation, 


$$
\sigma_{s c}\left(\vec{p}_{f}\right)=\vec{v}_{f}\left(\vec{p}_{f}\right) \cdot \vec{v}_{s}+\int d \vec{p}_{f}^{\prime} A^{c u r}\left(\vec{p}_{f}, \vec{p}_{f}^{\prime}\right) \Phi\left(\vec{p}_{f}^{\prime}\right) \sigma_{s c}\left(\vec{p}_{f}^{\prime}\right)
$$

Equations (34, 35, 38, 40, 18,24) are the basic equations used to calculate the temperature-dependent penetration depth for unconventional superconductors.

For a gap $\left|\Delta\left(\vec{p}_{f}\right)\right|$ with four-fold rotational symmetry about the $\hat{z}$-axis the resulting supercurrent is given by a diagonal superfluid density tensor with in-plane $\left(\rho_{s}^{\|}\right)$and z-axis $\left(\rho_{s}^{\perp}\right)$ superfluid densities given by

$$
\rho_{s}^{\|, \perp}=2 N_{f}\left(v_{f}^{\|, \perp}\right)^{2} \frac{\phi^{\|, \perp}}{1-\frac{1}{3} A^{\|, \perp} \phi \|, \perp},
$$

with

$$
\phi_{||}=\frac{3}{2} \int \frac{d \Omega}{4 \pi}\left(\hat{p}_{x}^{2}+\hat{p}_{y}^{2}\right) \Phi\left(\vec{p}_{f}\right), \quad \phi_{\perp}=3 \int \frac{d \Omega}{4 \pi} \hat{p}_{z}^{2} \Phi\left(\vec{p}_{f}\right) .
$$

Since $\phi_{\|, \perp} \sim \Delta_{0}^{2} \sim\left(1-T / T_{c}\right)$ near $T_{c}$, the Fermi liquid renormalizations of the penetration depth drop out near $T_{c}$, but may give substantial corrections to $\rho_{s}^{\|, \perp}(T)$ at low-temperatures. 34, 35]

\section{ZERO-FIELD PENETRATION DEPTH OF A $D_{X^{2}-Y^{2}}$ SUPERCONDUCTOR}

Consider the model of the $\mathrm{CuO}$ superconductors based on a $d_{x^{2}-y^{2}}$ order parameter. The general form of the order parameter is,

$$
\Delta\left(\vec{p}_{f}\right)=\Delta\left(\hat{p}_{x}^{2}-\hat{p}_{y}^{2}\right) * I\left(\vec{p}_{f}\right),
$$

where $I\left(\vec{p}_{f}\right)$ is invariant under the full point group, and $\hat{p}_{x, y}$ define the direction of the Fermi wavevector in the basal plane of the Fermi surface. Note that the nodes are required by the broken reflection symmetries. Two parameters determine the excitation spectrum in the clean limit; (i) the maximum value of $\left|\Delta\left(\vec{p}_{f}\right)\right|\left(=\Delta_{0}\right)$, and (ii) the angular slope of the gap near the node,

$$
\left.\mu \equiv \frac{1}{\Delta_{0}} \frac{d|\Delta(\vartheta)|}{d \vartheta}\right|_{\vartheta=\vartheta_{\text {node }}}
$$

where $\vartheta$ is the angle measured relative to one of the nodes (see Fig. 2).

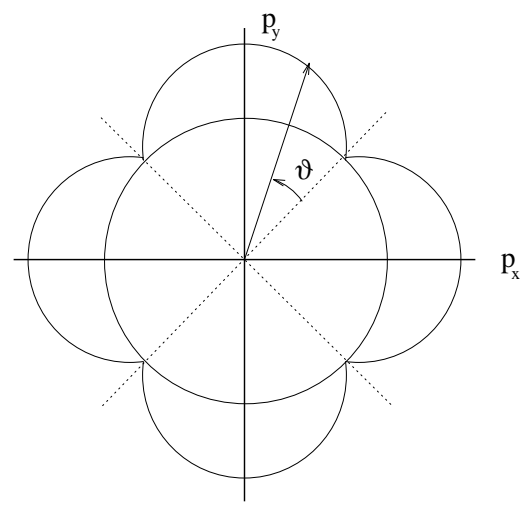

Fig. 2 Gap function for a $d_{x^{2}-y^{2}}$ superconductor. 
A simple two-parameter model for $\left|\Delta\left(\vec{p}_{f}\right)\right|$, which is useful for numerical calculations, is

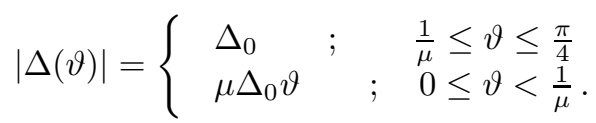

The maximum gap, for a fixed $\mu$, is obtained from a self-consistent solution to the gap equation. Figure 3 shows solutions of eq. (24) for $\Delta_{0}(T)$ as a function of temperature and impurity scattering. We obtain a gap ratio of $\Delta_{0} / T_{c}=1.9$ at $T=0$ for $\mu=2.7$ and $\Gamma=0$. Note that the leading temperature-dependent correction to the gap parameter for $T \ll T_{c}$ is $\delta \Delta_{0}(T) \sim T^{3}$, in the clean limit (appendix A).

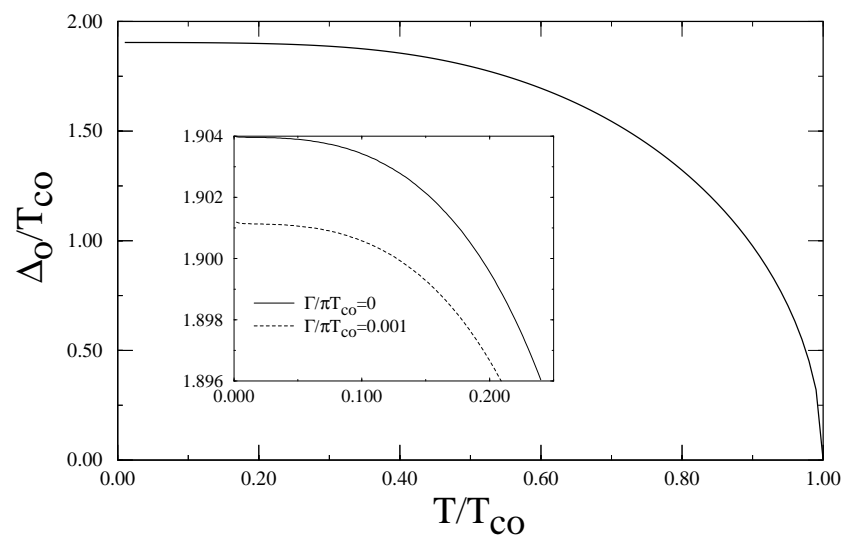

Fig. 3 Maximum gap for a $d_{x^{2}-y^{2}}$ superconductor as a function of temperature. The inset shows the $T^{3}$ deviation of $\Delta_{0}(T)$ at low-temperature.

The maximum gap is relatively insensitive to the angular slope of the gap near the nodes, except for small $\mu$, in which case the nodal region occupies a significant fraction of phase space. This behavior can be qualitatively understood by noting that the condensation energy at zero temperature is effectively determined by the strength of the pairing interaction, and therefore $T_{c}$. For an anisotropic gap $\Delta_{0}$ is enhanced to compensate for the regions of small gap. If we assume that the Fermi-surface average of $|\Delta(\vartheta)|^{2}$ is constant (fixed by $T_{c}$ ), then the maximum gap is given by $\Delta_{0}(\mu) \simeq 1.8 T_{c} /(1-8 / 3 \pi \mu)$, which is qualitatively the behavior obtained from the numerical solution to the gap equation shown in Fig. 4 . Note that the ususal one-parameter $d_{x^{2}-y^{2}}$ gap, $\Delta=\Delta_{0}\left(\hat{p}_{x}^{2}-\hat{p}_{y}^{2}\right)$, corresponds to $\mu=2$.

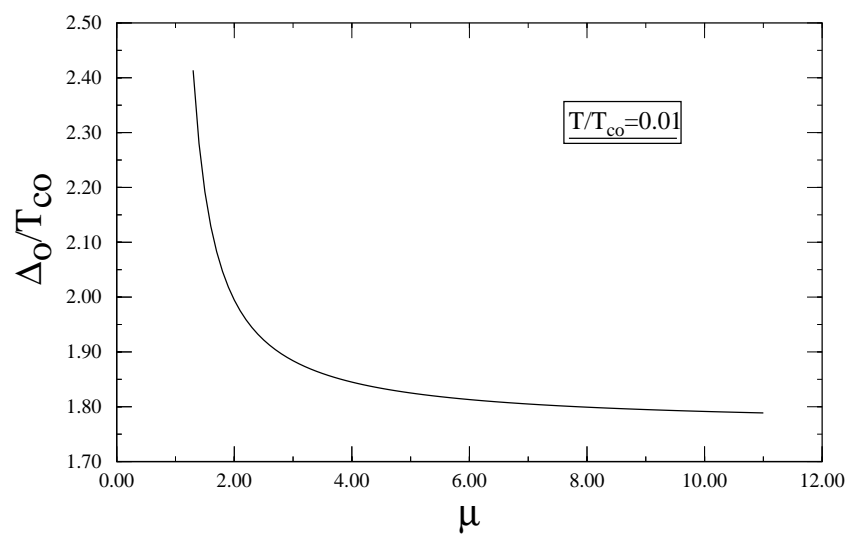

Fig. 4 Maximum gap for a $d_{x^{2}-y^{2}}$ superconductor as a function of the angular slope parameter, $\mu$. 
The suppression of $T_{c}$ by impurity scattering is determined by the pair-breaking parameter $\Gamma=\Gamma_{u} \sin ^{2} \delta_{0}$. Equation (23) for $T_{c}$ implies that unconventional pairing can be sensitive to impurity scattering, e.g. the superconducting transition is completely suppressed for $\left(\Gamma / \pi T_{c o}\right)_{c r i t}=\frac{1}{2} e^{-\gamma} \simeq 0.28$, which corresponds to an impurity mean-free path of $l_{\text {crit }}=v_{f} / 2 \Gamma \simeq 3.6 \xi_{0}$, where $\xi_{0}=v_{f} / 2 \pi T_{c 0}$ is the coherence length in the clean limit. The relatively small coherence length in the $\mathrm{CuO}$ superconductors is then an advantage for an unconventional order parameter. For weak pair-breaking, $\Gamma / 2 \pi T_{c} \ll 1$, the suppression of $T_{c}$ is given by, $\Delta T_{c} / T_{c 0}=-\pi \Gamma / 8 T_{c 0}$. Thus, for $C u O$ superconductors with $T_{c 0}=100 \mathrm{~K}$ and a suppression of less than $0.5 \mathrm{~K}$ we have $\Gamma<1.3 \mathrm{~K}$. For an in-plane coherence length of $\xi_{0}=14 \AA$ this corresponds to an impurity mean-free path, $l>3,450 \AA .2$

The magnitude of the gap parameter is also suppressed by impurity scattering. Figure 5 shows the suppression of $\Delta_{0}(0)$ as a function of $\Gamma$. Note that $\Delta_{0}(0)$, in contrast to $T_{c}$, is more strongly suppressed in the unitarity limit than in the Born limit for the same suppression of $T_{c}$.

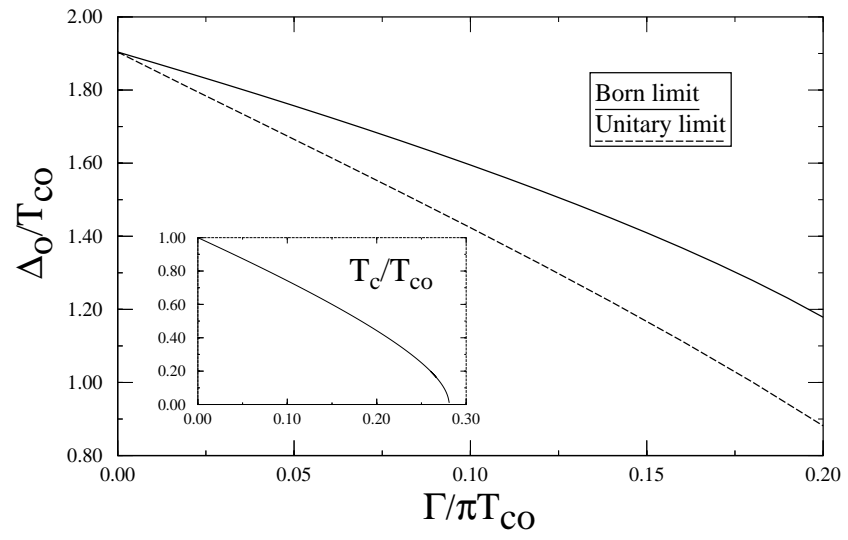

Fig. 5 Maximum gap at $T=0.01 T_{c}$ for a $d_{x^{2}-y^{2}}$ superconductor as a function of the impurity pair-breaking parameter. The inset shows the impurity pair-breaking effect on $T_{c}$.

In the clean limit the angle-resolved density of states, obtained from eq. (11), is given by the familiar BCS form,

$$
\mathcal{N}\left(\vec{p}_{f}, E\right)=\frac{|E|}{\sqrt{E^{2}-\left|\Delta\left(\vec{p}_{f}\right)\right|^{2}}} \Theta\left(E^{2}-\left|\Delta\left(\vec{p}_{f}\right)\right|^{2}\right) .
$$

For low energies, $|E| \ll \Delta_{0}$, the total density of states is dominated by the low-lying states near the nodes, and is linear in $|E|$. For the model gap function in eq.(45) the total density of states is

$$
\overline{\mathcal{N}}(E)=\int d \vec{p}_{f} \mathcal{N}\left(\vec{p}_{f}, E\right) \simeq \frac{2|E|}{\mu \Delta_{0}}, \quad|E|<\Delta_{0} .
$$

These low-lying excitations are responsible for the linear temperature dependence of the penetration depth for a superconductor with a line of nodes (or point nodes in 2D). Not surprisingly, the density of low-lying states near the nodes is determined by the angular slope of the gap; therefore, $\mu$ also determines the coefficient of the linear temperature dependence of the penetration depth (in the clean limit).

Although the small pair size in the cuprates leads to relatively weak suppression of $T_{c}$ from impurity scattering, the density of states at low-energy, $|E| \ll \Delta_{0}$, and therefore the leading temperature dependence of the penetration depth, are more sensitive to impurity scattering, particularly in the strong scattering limit. Figure 6 shows the density of states as a function of the scattering phase shift for fixed $\Gamma_{u}=0.1 \Delta_{0}$, corresponding to a rather high impurity

\footnotetext{
${ }^{2}$ For non-magnetic, s-wave impurities in $2 \mathrm{D}$, the impurity resistivity is given by $\rho_{i m p}^{-1}=e^{2} N_{f} v_{f}^{2} / 4 \Gamma$, where $\Gamma$ is the same pairbreaking parameter that enters the Abrikosov-Gorkov formula. Thus, we can express $T_{c} / T_{c 0}$ as a function of $\rho_{i m p}$, independent of the scattering phase shift, $\delta_{0}$. However, inelastic scattering, which is important at $T \sim T_{c}$, destroys this simple result. [36.37]
} 
concentration. The modification of the density of states at low-energy is negligible in the Born limit $\left(\delta_{0}=\pi / 20\right)$ and remains essentially linear even for intermediate phase shifts $\left(\delta_{0}=\pi / 4\right)$. However, as the strength of the scattering increases a finite density of states at $E=0$ develops, becoming of order $\overline{\mathcal{N}}(0) \simeq 0.4$ in the unitarity limit. In addition, $\overline{\mathcal{N}}(E)$ deviates from linearity below $E \simeq 0.4 \Delta_{0}$. This is the cross-over energy scale, below which impurity scattering strongly modifies the low-energy spectrum. The cross-over energy, $\varepsilon^{*}$, can be calculated from the lowest energy scale for the renormalized Matsubara frequency $\tilde{\epsilon}_{n}$ as $T \rightarrow 0$. From eq. (18) the cross-over scale at $T=0$ is given by

$$
2 \tau=\frac{1}{\sigma \Gamma_{u}}=\frac{\mathcal{D}\left(\varepsilon^{*}\right)}{(1-\sigma)+\sigma\left(\varepsilon^{*} \mathcal{D}\left(\varepsilon^{*}\right)\right)^{2}},
$$

where $\sigma=\sin ^{2} \delta_{0}$, and $\mathcal{D}\left(\varepsilon^{*}\right)$ can be calculated to leading logarithmic accuracy $\left(\ln \left(\Delta_{0} / \varepsilon^{*}\right) \gg 1\right)$ for the $d_{x^{2}-y^{2}}$ state,

$$
\mathcal{D}\left(\varepsilon^{*}\right) \simeq \frac{4}{\pi \mu \Delta_{0}} \ln \left(2 \Delta_{0} / \varepsilon^{*}\right)
$$

In the unitarity limit this scale can be a sizable fraction of $\Delta_{0}$ even in the dilute limit, $\varepsilon^{*} \sim \sqrt{\Gamma_{u} \Delta_{0}}$, but in the Born limit the cross-over scale is exponentially small, $\varepsilon^{*} \sim 2 \Delta_{0} \exp \left(-\mu \pi \tau \Delta_{0} / 2\right)$. For more detailed discussions of the density of states of d-wave superconductors see Refs. ( [38,39]).

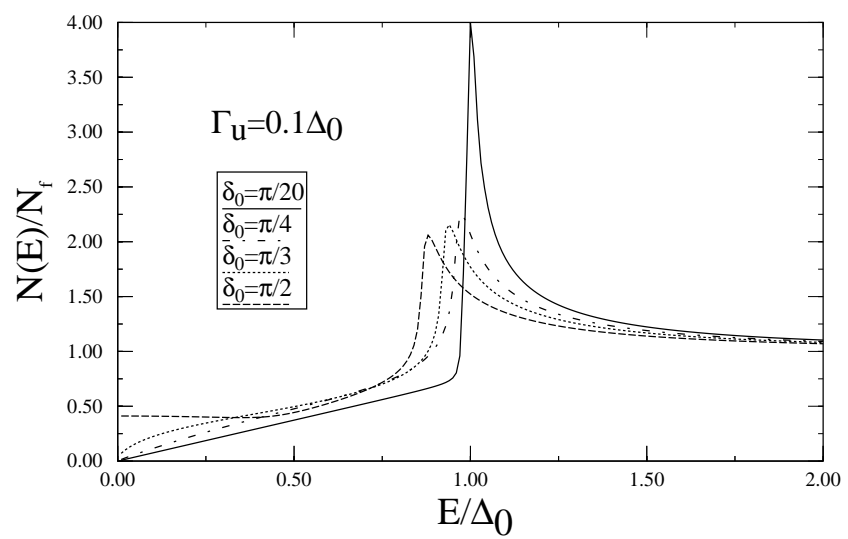

Fig. 6 Density of states vs. scattering phase shift. The impurity concentration is fixed with $\Gamma_{u} / \Delta_{0}=0.1$. Note the finite density of states and the suppression of the maximum gap in the unitarity limit.

\section{Penetration depth for the $d_{x^{2}-y^{2}}$ gap}

The temperature dependence of the penetration depth for a $d_{x^{2}-y^{2}}$ gap function is obtained from eq.(34) (neglecting Fermi liquid corrections). We assume a cylindrical Fermi surface and a gap function parametrized by $\mu$ and $\Delta_{0}$ as in eq. (45). The in-plane penetration depth becomes

$$
\frac{1}{\lambda_{\|}^{2}}=\frac{4 \pi e^{2} N_{f}\left(v_{f}^{\|}\right)^{2}}{c^{2}} \oint \frac{d \vartheta}{2 \pi} \pi T \sum_{\epsilon_{n}} \frac{|\Delta(\vartheta)|^{2}}{\left[Z\left(\epsilon_{n}\right)^{2} \epsilon_{n}^{2}+|\Delta(\vartheta)|^{2}\right]^{3 / 2}}
$$

where the integration is over the Fermi circle in the basal plane, and $Z\left(\epsilon_{n}\right)$ is the impurity renormalization factor for s-wave scattering centers. In the clean limit the leading correction to the penetration depth at low-temperatures is linear in $T$, typical of a gap with a line of nodes, 35.4042

$$
\frac{\delta \lambda_{\|}(T)}{\lambda_{\|}(0)}=\left(\frac{2 \ln 2}{d|\Delta(\vartheta)| /\left.d \vartheta\right|_{\text {node }}}\right) T+\ldots \quad ; \quad T \ll T_{c},
$$

where $d|\Delta(\vartheta)| /\left.d \vartheta\right|_{\text {node }}$ is the angular slope of $|\Delta(\vartheta)|$ at the node. For the model gap in eq. 45$), d|\Delta(\vartheta)| /\left.d \vartheta\right|_{\text {node }}=$ $\mu \Delta_{0}$. 
The report by Hardy, et al. [1] of a linear temperature dependence to $\delta \lambda_{||}(T)$ for single crystals of $\mathrm{YBa}_{2} \mathrm{Cu}_{3} \mathrm{O}_{6.95}$ provided the first substantial evidence for a superconducting state with a line of nodes in the excitation gap. Figure 7 shows the low-temperature data for $\delta \lambda_{\|}(T)$ reported in Ref.( 443$\left.]\right)$. The solid line is a calculation of the penetration depth for a $d_{x^{2}-y^{2}}$ gap with the angular slope adjusted to fit the data for $T<20 \mathrm{~K}$. For the absolute penetration depth we assume $\lambda_{\|}(0)=1,400 \AA$. [44] The slope of the penetration depth at low temperature is $4.3 \AA / K$, and the fit to eq.(51) gives $\mu=2.7, \Delta_{0}(0) / T_{c}=1.9$, and $d|\Delta(\vartheta)| /\left.d \vartheta\right|_{\text {node }}=\mu \Delta_{0}(0) \simeq 5.1 T_{c}$. For comparison, the one-parameter $d_{x^{2}-y^{2}}$ model, $\Delta=\Delta_{0} \cos (2 \phi)$ with $\Delta_{0}$ calculated self-consistently, gives $d|\Delta(\vartheta)| /\left.d \vartheta\right|_{\text {node }}=\mu \Delta_{0}(0)=4.3 T_{c}$.

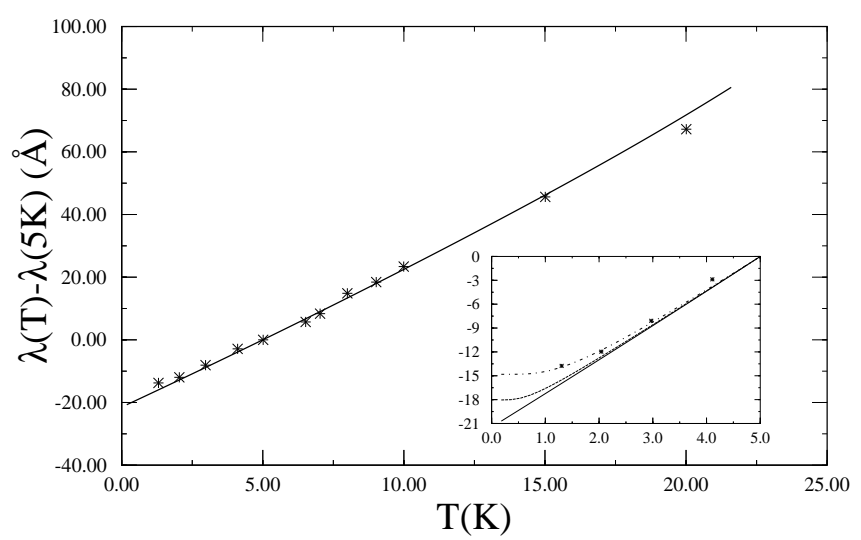

Fig. 7 Penetration depth as a function of temperature. The $(*)$ are experimental data obtained from Ref. ( [1]) the solid line is the best fit with $\mu \simeq 2.7, \Delta_{0} / T_{c} \simeq 1.9$. The inset shows the effect of a tiny gap at the nodal positions on the temperature dependence of the penetration depth. The axes are the same as those of the main graph. The middle (top) curve in the inset corresponds to a tiny gap of $\Delta_{\min } / \Delta_{0}=1 \%(2 \%)$.

Also shown in Fig. 7 is the effect of a tiny gap at the nodal positions. Tiny gaps can arise in strongly anisotropic conventional superconductors, 45] or if the unconventional order parameter contains a small component of another representation. For example, an order parameter of the form $d_{x^{2}-y^{2}}+i \sqrt{\varepsilon} d_{x y}$ has a gap function, $\left|\Delta\left(\vec{p}_{f}\right)\right|=\Delta_{0}\left[\cos ^{2}(2 \phi)+\varepsilon \sin ^{2}(2 \phi)\right]^{1 / 2}$, which is strongly anisotropic for small $\varepsilon$ with a tiny gap of order $\varepsilon \Delta_{0}$ near the nodes of the $d_{x^{2}-y^{2}}$ component. [46] The data of Ref. ( [1]) implies that $\varepsilon<2 \%$.

Precision measurements of the penetration depth in thin films show a $T^{2}$ behavior at low temperatures rather than the linear temperature dependence characteristic of a $d_{x^{2}-y^{2}}$ order parameter. [47 52, 12] This difference may be due to scattering by a higher concentration of defects present in the films. Hirschfeld and Goldenfeld [42] argue that the $T^{2}$ dependence in films and the $T$ dependence of $\delta \lambda_{\|}(T)$ reported for single crystals can be understood within the same $d_{x^{2}-y^{2}}$ model for the pairing state provided the films are relatively dirty compared to the single crystals. However, impurity scattering or defect scattering is pair-breaking in unconventional superconductors, so in order to explain the weak or negligible suppression of $T_{c}$ in films (compared to $T_{c}$ in the single crystals of Ref.( [1]), the authors of Ref. ( 42]) argue that the scattering responsible for the $T^{2}$ dependence of $\delta \lambda_{\|}(T)$ results from a dilute concentration of strong scattering centers with phase shifts near the unitarity limit. A small concentration of unitarity scatterers leads to strong modification of the density of states in the small phase space region near the nodes. Since $\delta \lambda_{\|}$is determined by these low-energy excitations, the temperature dependence of $\delta \lambda_{\|}(T)$ for $T \ll T_{c}$ may be strongly modified even when the suppression of $T_{c}$ by a dilute concentration of scatterers is negligible.

In the Born limit (weak scattering) the density of states, even near the nodes is nearly unchanged. Thus, a much higher concentration of defects is needed to generate $\delta \lambda_{\|}(T) \sim T^{2}$ for $T \lesssim 0.2 T_{c}$, which is accompanied by a sizeable suppression of $T_{c}$. The sensitivity of the low-energy excitation spectrum to the scattering strength is reflected in the temperature dependence of the penetration depth shown in Fig. 8. The concentration of s-wave scatterers is fixed and the curves show the evolution from a linear $T$ dependence in the Born limit $\left(\delta_{0}=\pi / 20\right)$, and intermediate phase shifts, to the $T^{2}$ dependence in the unitarity limit $\left(\delta_{0}=\pi / 2\right)$. Note that the cross-over from $\delta \lambda_{\|} \sim T$ to $\delta \lambda_{\|} \sim T^{2}$ is abrupt, occuring very near the unitarity limit for dilute concentrations. A sharp cross-over in the excitation spectrum as a function of phase shift has also been noted by Preosti, et al. [38]. Thus, it is worth emphasizing that for dilute point impurities it is not merely 'strong scattering' that is required to obtain $\delta \lambda_{\|} \sim T^{2}$ with minimal reduction in $T_{c}$, but scattering with $\delta_{0} \rightarrow \pi / 2$. 


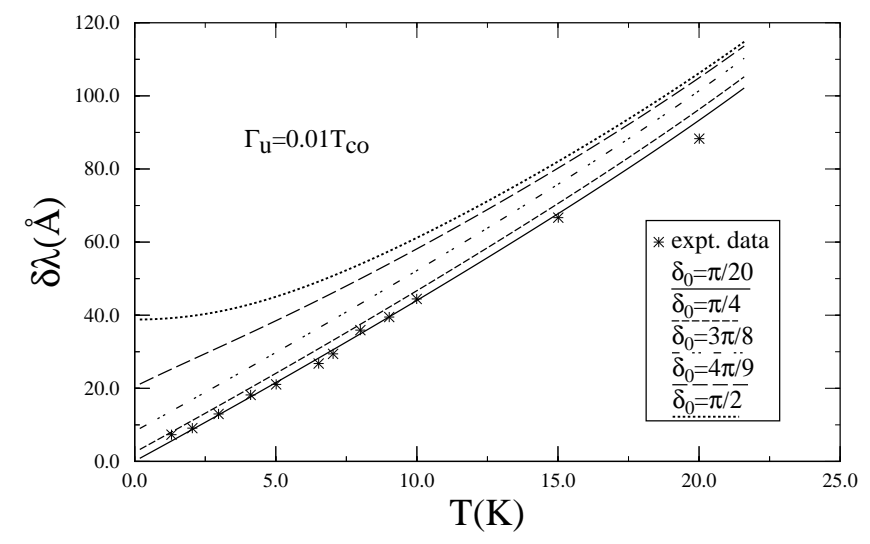

Fig. 8 Penetration depth as a function of temperature for phase shifts ranging from the Born limit to the unitarity limit. The concentration is fixed with $\Gamma_{u}=0.01 T_{c}$.

In the unitarity limit even dilute concentrations of impurities can strongly modify the low-energy spectrum. In the unitarity limit the cross-over energy scale is $\varepsilon^{*} \sim \sqrt{\Gamma_{u} \Delta_{0}}$. Thus, $\delta \lambda_{\|}(T)$ deviates from the linear $T$ dependence for $T<\varepsilon^{*}$. The sensitivity of $\delta \lambda_{\|}(T)$ at $T \ll T_{c}$ for unitarity scattering (see Fig. 9) places a strong constraint on the concentration of scatterers that can be present in clean single crystals that shows $\delta \lambda_{\|}(T) \sim T$ down to low temperatures. For the data of Ref. ( [ [] $)$ we find $\Gamma_{u} / \Delta_{0} \lesssim 0.0001$ given that $\delta \lambda_{\|}(T) \sim T$ down to $T \simeq 1 K$.

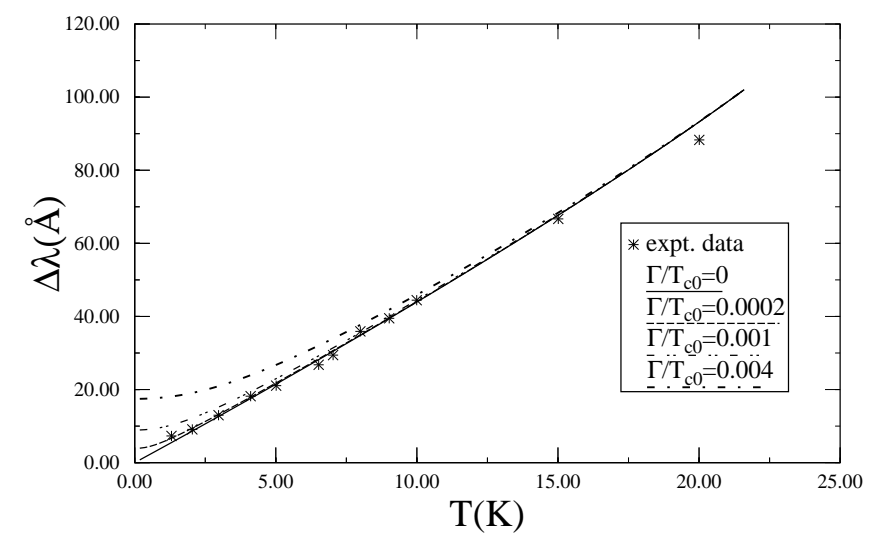

Fig. 9 Penetration depth as a function of temperature and impurity concentration $\left(\sim \Gamma_{u}\right)$ in the unitarity scattering limit.

\section{NONLINEAR CURRENT RESPONSE}

In the Meissner geometry the screening current is proportional to the applied surface field, $j_{s} \sim c H / \lambda$. As $H$ is increased nonlinear field corrections to the constitutive equation for the supercurrent may become significant. In conventional type II superconductors nonlinear corrections to the current-velocity relation arise from the thermal population of quasiparticles, and vortex nucleation generally occurs before these nonlinear effects become important.

In unconventional superconductors with nodes in the excitation gap the nonlinear field correction to the supercurrent is substantially larger than in conventional superconductors with other similar material properties. The origin of the anomalous nonlinear Meissner effect is the contribution to the screening current associated with the quasiparticle 
states near the nodal lines. As a result the nonlinear Meissner effect may be used to detect the nodal structure of the gap of an unconventional superconductor. [15]

In the limit $\left|\vec{v}_{f} \cdot \vec{\nabla}\right| \Delta \| / \pi T_{c} \sim \xi / \lambda \ll 1$ the current can be expressed as a local function of the condensate velocity, $\vec{v}_{s}(\vec{R})$. In the presence of a condensate flow the local solution to the transport equation, $\hat{g}\left(\vec{p}_{f}, \vec{R} ; \epsilon_{n}\right)$, is given by eq. (11), but evaluated with $i \tilde{\epsilon}_{n} \rightarrow i \tilde{\epsilon}_{n}-\sigma_{v}$, where $\sigma_{v}\left(\vec{p}_{f}, \vec{R}\right)=\vec{v}_{f} \cdot \vec{v}_{s}(\vec{R})$. The current response obtained from eq. (3) is

$$
\vec{j}_{s}=-2 e N_{f} \int d \vec{p}_{f} \vec{v}_{f}\left(\vec{p}_{f}\right) \pi T \sum_{\epsilon_{n}} \frac{\sigma_{v}\left(\vec{p}_{f}\right)-i \tilde{\epsilon}_{n}}{\sqrt{\left(\tilde{\epsilon}_{n}+i \sigma_{v}\left(\vec{p}_{f}\right)\right)^{2}+\left|\tilde{\Delta}\left(\vec{p}_{f} ; \epsilon_{n}\right)\right|^{2}}} .
$$

One point to note in calculating the density of states, or current, at finite flow with impurity scattering is that the impurity-renormalization of $\Delta\left(\vec{p}_{f}\right)$ need not vanish, even for s-wave impurity scattering and $\left\langle\Delta\left(\vec{p}_{f}\right)\right\rangle=0$. The reason is clear from eq. $(12)$ for $\tilde{\Delta}\left(\vec{p}_{f} ; \epsilon_{n}\right)$; the kernel no longer vanishes by symmetry with the replacement, $\tilde{\epsilon}_{n} \rightarrow \tilde{\epsilon}_{n}+i \vec{v}_{f} \cdot \vec{v}_{s}$ for a general flow field. However, for special directions of $\vec{v}_{s}$, e.g. $\vec{v}_{s}$ parallel to a node, the impurity correction to $\Delta\left(\vec{p}_{f}\right)$ vanishes, $\tilde{\Delta}=\Delta\left(\vec{p}_{f}\right)$.

Equation (52) for the current can be transformed by contour integration and analytic continuation to the real axis to give

$$
\vec{j}_{s}=-2 e N_{f} \int_{\sigma_{v}>0} d \vec{p}_{f} \vec{v}_{f}\left(\vec{p}_{f}\right) \int_{-\infty}^{+\infty} d E f(E)\left[\mathcal{N}_{+}\left(\vec{p}_{f}, E\right)-\mathcal{N}_{-}\left(\vec{p}_{f}, E\right)\right],
$$

where $\mathcal{N}_{ \pm}\left(\vec{p}_{f}, E\right)$ is the density of states for quasiparticles that are co-moving $\left(+\vec{v}_{f} \cdot \vec{v}_{s}>0\right)$ and counter-moving $\left(-\vec{v}_{f} \cdot \vec{v}_{s}<0\right)$ relative to the condensate flow. The integral is taken over the half space $\sigma_{v}=\vec{v}_{f} \cdot \vec{v}_{s}>0$ with the counter-moving excitations included by inversion symmetry: $\mathcal{C}_{i} \sigma_{v}=-\sigma_{v}$ and $\left|\Delta\left(\mathcal{C}_{i} \vec{p}_{f}\right)\right|=\left|\Delta\left(\vec{p}_{f}\right)\right|$. This result is general enough to cover nonlinear field corrections to the current for superconductors with an unconventional order parameter and pair-breaking effects from impurity scattering.

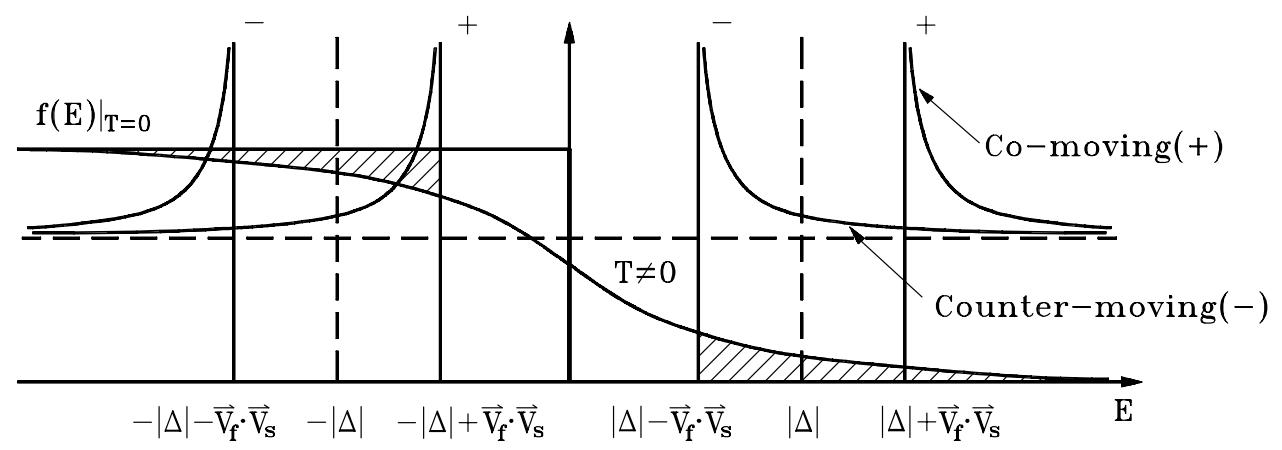

Fig. 10 Density of states for co-moving $\left(+\vec{v}_{f} \cdot \vec{v}_{s}\right)$ and counter-moving $\left(-\vec{v}_{f} \cdot \vec{v}_{s}\right)$ excitations at a point $\vec{p}_{f}$ on the Fermi-surface where $\left|\vec{v}_{f} \cdot \vec{v}_{s}\right|<\left|\Delta\left(\vec{p}_{f}\right)\right|$.

The difference in the nonlinear current-velocity relation for conventional and unconventional order parameters appears in the contributions to the current from the co-moving and counter-moving excitation spectrum at $T=0$. The spectrum is shown Fig. 10 in the clean limit for a specific direction $\vec{p}_{f}$ in which $\vec{v}_{f} \cdot \vec{v}_{s}<\left|\Delta\left(\vec{p}_{f}\right)\right|$. At zero temperature only the co-moving and counter-moving quasiparticle states with $E<0$ contribute to the current.

\section{Nonlinear current: conventional gap}

For a conventional superconductor with an isotropic gap at $T=0$ the current is easily calculated from the difference in the number of co-moving versus counter-moving quasiparticles that make up the condensate,

$$
\vec{j}_{s}=-2 e N_{f} \int_{\sigma_{v}>0} d \vec{p}_{f} \vec{v}_{f}\left(\vec{p}_{f}\right)\left[2 \vec{v}_{f} \cdot \vec{v}_{s}\right]=-e \rho \vec{v}_{s} \quad, \quad v_{s}<v_{f} / \Delta_{0},
$$

with $\rho=N_{f} v_{f}^{2}$ for a cylindrical Fermi surface. The main point is that the current is linear in $\vec{v}_{s}$ for velocities up to the bulk critical velocity, $v_{c}=v_{f} / \Delta_{0}$. At $v_{s}=v_{c}$ the edge of the spectrum for the upper branch $(E>0$ for 
$\left.v_{s}<v_{c}\right)$ of counter-moving excitations drops below $E=0$, and the edge of the spectrum for the lower branch $(E<0$ for $v_{s}<v_{c}$ ) of co-moving excitations shifts above $E=0$. As a result the current carried by the condensate drops rapidly above the critical velocity. The current is nonanalytic at $v_{c}$ because a branch of counter-moving (co-moving) excitations that are unoccupied (occupied) for $v_{s}<v_{c}$ become occupied (unoccupied) $v_{s}>v_{c}$. For example, for a 1D Fermi surface the current becomes, $j_{s}=-2 e N_{f} v_{f}^{2}\left\{v_{s}-\Theta\left(v_{s}-v_{c}\right) \sqrt{v_{s}^{2}-v_{c}^{2}}\right\}$.

At non-zero temperatures thermal occupation of the upper branches and de-population of the lower branches reduces the condensate supercurrent. In the clean limit eq. 53 can be transformed to

$$
\begin{gathered}
\vec{j}_{s}=-2 e N_{f} \int d \vec{p}_{f} \vec{v}_{f}\left(\vec{v}_{f} \cdot \vec{v}_{s}\right)+\vec{j}_{q p}, \\
\vec{j}_{q p}=-4 e N_{f} \int_{0}^{\infty} d \xi \int d \vec{p}_{f} \vec{v}_{f}\left[f\left(\sqrt{\xi^{2}+|\Delta|^{2}}+\vec{v}_{f} \cdot \vec{v}_{s}\right)\right],
\end{gathered}
$$

which separates the condensate contribution to the current, the fully occupied negative energy branches shown in Fig. 10 , from $\vec{j}_{q p}$, the current carried by the excitations associated with population of the upper branches and depopulation of the lower branches in Fig. 10. The current carried by the excitations is a backflow current. For low velocities the net current is linear in $\vec{v}_{s}, \vec{j}_{s}=-e \rho_{s}(T) \vec{v}_{s}$, where $\rho_{s}(T)$ is the superfluid density in the two-fluid model,

$$
\rho_{s}(T)=\rho-N_{f} v_{f}^{2} \int_{0}^{\infty} d \xi \frac{\operatorname{sech}^{2}\left(\sqrt{\xi^{2}+|\Delta|^{2}}\right)}{2 T} .
$$

For larger flow velocities the linear relation breaks down. The leading correction to the current-velocity relation for $v_{s}<v_{c}$ is,

$$
\vec{j}_{s}=-e \rho_{s}(T) \vec{v}_{s}\left[1-\alpha(T)\left(\frac{v_{s}}{v_{c}}\right)^{2}\right] .
$$

The nonlinear correction is third-order in $v_{s}$ and determined by the coefficient $\alpha(T) \geq 0$ and the bulk critical velocity, $v_{c}=\Delta(T) / v_{f}$. There are two sources to the nonlinear current. At finite $v_{s}$ there is a difference in the thermal occupations of co-moving and counter-moving excitations. Since the counter-moving branch of has a larger occupation than the co-moving branch $\left(f\left(E-v_{f} v_{s}\right)\right.$ compared to $\left.f\left(E+v_{f} v_{s}\right)\right)$, the thermal excitations further reduce the current compared with the linear response value. In addition the condensate velocity is also pairbreaking, i.e. $v_{s}$ reduces the magnitude of the mean-field gap parameter, further reducing the current density at finite flow. Figure 11 shows the temperature dependence of the nonlinear correction to eq.(58). Note that $\alpha(T) \sim \exp (-\Delta / T)$ for $T \rightarrow 0$. There are no excitations that contribute to the backflow current at $T=0$ and the constitutive equation is strictly linear for velocities below the bulk critical velocity, $v_{c}=\Delta / v_{f}$.

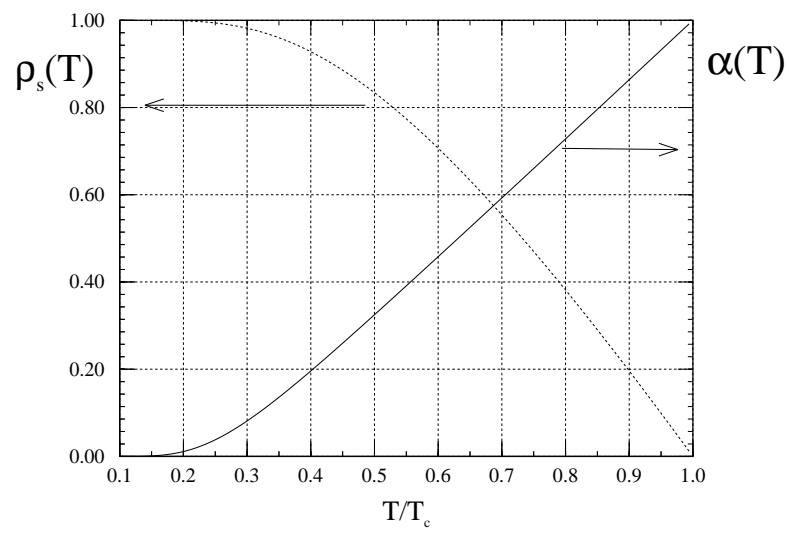

Fig. 11 Temperature dependence of $\rho_{s}(T)$ and the nonlinear coefficient $\alpha(T)$ for an s-wave gap. 
The relevance of the nonlinear current-velocity constitutive equation to the penetration of magnetic fields into a superconductor is qualitatively clear. The reduction of the current by the flow reduces the effective superfluid density, and, therefore, increases the penetration of the field into the superconductor. Since the current is proportional to the field in linear order, the leading correction to the effective penetration length is quadratic in the surface field. Solution of the nonlinear London equation in the Meissner geometry gives the following result for the field-dependence of the penetration depth (see appendix B),

$$
\frac{1}{\lambda(T, H)}=\frac{1}{\lambda(T)}\left\{1-\frac{3}{4} \alpha(T)\left[\frac{H}{H_{0}(T)}\right]^{2}\right\}
$$

where $1 / \lambda(T)^{2}=8 \pi e^{2} \rho_{s}(T) / 3 c^{2}$ is the zero-field London penetration depth, and $H_{0}(T)=e \lambda(T) / c v_{c}(T)$ is of order the thermodynamic critical field. Thus, in the London limit nonlinear Meissner effect in a conventional superconductor is exponentially small at low temperatures and is quadratic in $H / H_{0}$.

\section{Nonanalytic supercurrents at $\mathbf{T}=0: d_{x^{2}-y^{2}}$ gap}

The expansion of the current in $\vec{v}_{s}$ breaks down for an unconventional superconductor with nodes in the gap. This is clear from eq. (52); a Taylor expansion in $\sigma_{v}$ fails for $T \ll T_{c}$ when there are directions $\vec{p}_{f}$ where $\left|\Delta\left(\vec{p}_{f}\right)\right|$ and $\left|\tilde{\epsilon}_{n}\right|$ are always small compared to $\left|\sigma_{v}\left(\vec{p}_{f}\right)\right|$. In the clean limit for a $d_{x^{2}-y^{2}}$ gap the breakdown of the Taylor expansion leads to a nonanalytic current-velocity relation at $T=0$ of the form $\vec{j}_{s}=-e \rho \vec{v}_{s}\left\{1-\left|\vec{v}_{s}\right| / v_{0}\right\}$, where $v_{0} \sim \Delta_{0} / v_{f}$. ${ }^{3}$ The physical origin of this nonanalytic current is easily understood by considering the $d_{x^{2}-y^{2}}$ gap with $\vec{v}_{s}$ directed along a node, as shown in Fig. 12 (left panel).
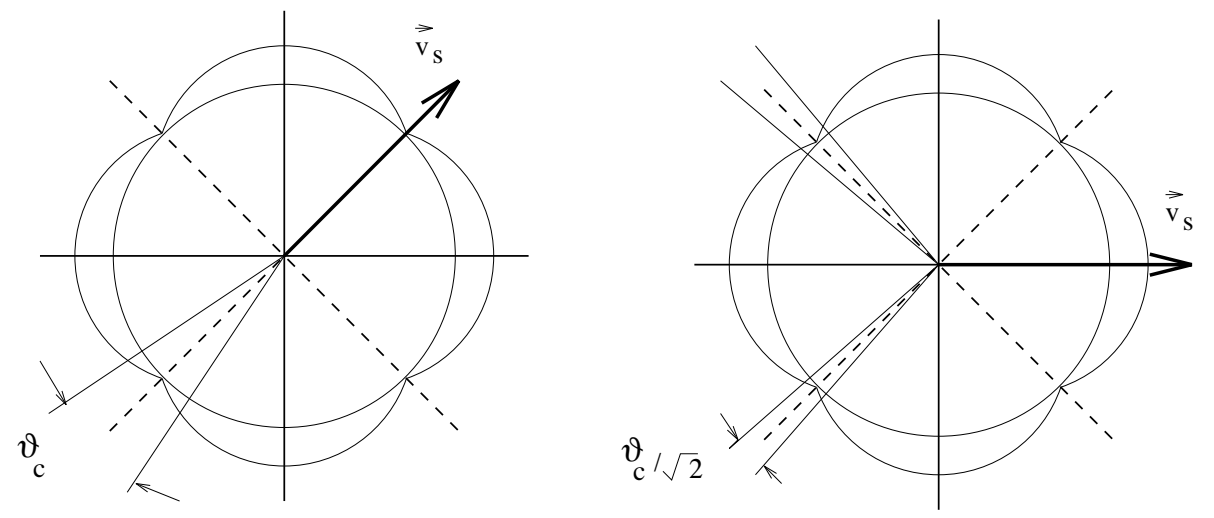

Fig. 12 Phase space contributing to the quasiparticle backflow jets at $T=0$ for $\vec{v}_{s} \|$ node, and $\vec{v}_{s} \|$ antinode.

For any $v_{s} \neq 0$ there is a region of the Fermi surface with $\left|\Delta\left(\vec{p}_{f}\right)\right|+\vec{v}_{f} \cdot \vec{v}_{s}<0$, in which the upper branch of the counter-moving excitations (see Fig. 10) have negative energy and become populated. The non-analytic dependence on $\vec{v}_{s}$ reflects the occupation of this counter-moving branch of excitations at $T=0$. Figure 12 illustrates the phase space contributing to the backflow current. For $\left|v_{f} v_{s}\right| \ll \Delta_{0}$ the wedge of occupied states is $-\vartheta_{c} \leq \vartheta \leq \vartheta_{c}$, with $\vartheta_{c}=v_{f}^{*} v_{s} / \mu \Delta_{0}$, where $v_{f}^{*}$ is the Fermi velocity at the node and $\mu \Delta_{0}$ is the angular slope of $|\Delta(\vartheta)|$ at the node. The current is calculated by transforming eq. (53) to

$$
\vec{j}_{s}=-2 e N_{f} \int d \vec{p}_{f} \vec{v}_{f}\left[\left(\vec{v}_{f} \cdot \vec{v}_{s}\right)+2 \int_{0}^{\infty} d \xi f\left(E(\xi)+\vec{v}_{f} \cdot \vec{v}_{s}\right)\right],
$$

\footnotetext{
${ }^{3}$ Nonanalytic currents have been investigated in superfluid ${ }^{3} \mathrm{He}-\mathrm{A}$, initially by Volovik and Mineev. [53] This work is closely related to a number of theoretical investigations of the hydrodynamical equations of superfluid ${ }^{3} \mathrm{He}$ in the limit $T \rightarrow 0$ (see Ref. ( 54]). For an analysis of the non-analytic current in ${ }^{3} \mathrm{He}-\mathrm{A}$ see Ref. ( 55]); these authors also calculate the non-analytic current-velocity relation for an axially symmetric, polar state with $\Delta \sim \hat{p}_{z}$. The polar model is examined in more detail by Choi and Muzikar. 56
} 
where $E(\xi)=\sqrt{\xi^{2}+\left|\Delta\left(\vec{p}_{f}\right)\right|^{2}}$. The first term is the condensate current, $-e \rho \vec{v}_{s}$. The backflow current at $T=0$ is easily calculated from the phase space of occupied counter-moving excitations. With the velocity directed along the nodal line $\hat{p}_{x}=\hat{p}_{y}$, i.e. $\vec{v}_{s}=v_{s} \hat{x}^{\prime}$ as shown in Fig. 12 (left panel), the occupied states give

$$
\begin{array}{r}
\vec{j}_{q p}=-2 e N_{f} \int d \vec{p}_{f} \vec{v}_{f} \Theta\left(-\vec{v}_{f} \cdot \vec{v}_{s}-\left|\Delta\left(\vec{p}_{f}\right)\right|\right) \sqrt{\left(\vec{v}_{f} \cdot \vec{v}_{s}\right)^{2}-\left|\Delta\left(\vec{p}_{f}\right)\right|^{2}} \\
=-2 e N_{f} v_{f}^{*} \int_{-\vartheta_{c}}^{\vartheta_{c}} \frac{d \vartheta}{2 \pi} \sqrt{\left(v_{f}^{*} v_{s}\right)^{2}-\left(\mu \Delta_{0} \vartheta\right)^{2}}\left(-\hat{x}^{\prime}\right) .
\end{array}
$$

To leading order in $\left(v_{f}^{*} v_{s} / \Delta_{0}\right)$ we obtain a total current of

$$
\vec{j}_{s}=-e \rho \vec{v}_{s}\left\{1-\frac{\left|\vec{v}_{s}\right|}{v_{0}}\right\} \quad\left(\vec{v}_{s}|| \text { node }\right)
$$

where $v_{0}=\mu \Delta_{0} / v_{f}^{*}$ is of order the bulk critcial velocity scale. Equation (62) clearly holds for $\vec{v}_{s}$ directed along any of the four nodes. Note that the current is parallel to the velocity, and that the counter-moving excitations reduce the supercurrent, as expected. Also the nonlinear correction is quadratic rather than cubic, as is obtained for the conventional gap, and with the characteristic scale determined by $v_{0}=\mu \Delta_{0} / v_{f}^{*}$.

Unlike the linear response current, the nonlinear quasiparticle current is anisotropic in the basal plane. A velocity field directed along the maximum direction of the gap (antinode), $\vec{v}_{s}=v_{s} \hat{x}$, produces two counter-moving jets, albeit with reduced magnitude because the projection of $\vec{v}_{s}$ along the nodal lines is reduced by $1 / \sqrt{2}$ (Fig. 12, right panel). The critical angle defining the occupied states in this case is given by $\frac{1}{\sqrt{2}}\left(v_{s} / v_{0}\right)$. The resulting current is easily calculated to be

$$
\vec{j}_{q p}=-e \rho\left[\frac{v_{s}}{\sqrt{2}}\left(\frac{v_{s} / \sqrt{2}}{v_{0}}\right)\left(-\hat{x}^{\prime}\right)+\frac{v_{s}}{\sqrt{2}}\left(\frac{v_{s} / \sqrt{2}}{v_{0}}\right)\left(+\hat{y}^{\prime}\right)\right],
$$

giving a total current of

$$
\vec{j}_{s}=-e \rho \vec{v}_{s}\left\{1-\frac{1}{\sqrt{2}} \frac{\left|\vec{v}_{s}\right|}{v_{0}}\right\} \quad\left(\vec{v}_{s}|| \text { antinode }\right)
$$

which is again parallel to the velocity and has a quadratic nonlinear correction. However, the magnitude of the nonlinear term is reduced by $1 / \sqrt{2}$. This anisotropy is due to the relative positions of the nodal lines and is insensitive to the details of the anisotropy of the the Fermi surface or Fermi velocity because the quasiparticle states that contribute to the current, for either orientation of the velocity, are located in a narrow wedge, $\vartheta \leq \vartheta_{c} \sim\left(v_{s} / v_{o}\right) \ll 1$, near the nodal lines. Thus, the occupied quasiparticle states near any of the nodes have essentially the same Fermi velocity and density of states; only the relative occupation of the states is modified by changing the direction of the velocity.

The dependence of the supercurrent on the positions of the nodal lines in momentum space suggests that the anisotropy can be used to distinguish different unconventional gaps with nodes located in different directions in momentum space. For example, the $d_{x y}$ state $\left(\mathrm{B}_{2 g}\right.$ representation), $\Delta \sim \hat{p}_{x} \hat{p}_{y}$, would also exhibit a four-fold anisotropy, but the nodal lines are rotated by $\pi / 4$ relative to those of the $d_{x^{2}-y^{2}}$ state. The order parameter $\Delta \sim$ $\hat{p}_{x} \hat{p}_{y}\left(\hat{p}_{x}^{2}-\hat{p}_{y}^{2}\right)$, corresponding to the $A_{2 g}$ representation, would exhibit a more complicated $2 \times 4$-fold anisotropy.

Anisotropy in the in-plane current implies a similar anisotropy in the field dependence of the in-plane penetration length. Consider the geometry in which the superconductor occupies the half-space $z>0$, with $z \| \hat{c}$. For a surface field $\vec{H}$ directed along a nodal line, Maxwell's equation combined with eq. (62) for the current and the gauge condition $\vec{\nabla} \cdot \vec{v}_{s}=0$ reduces to

$$
-\frac{\partial^{2} v_{s}}{\partial z^{2}}=\frac{4 \pi e^{2}}{c^{2}} j_{s}\left[\vec{v}_{s}\right]=-\frac{v_{s}}{\lambda_{\|}^{2}}\left\{1-\frac{\left|v_{s}\right|}{v_{0}}\right\}
$$

We define the effective penetration length in terms of the static surface impedance, $1 / \lambda_{\|}=-\left.(1 / H)(\partial b / \partial z)\right|_{z=0}$. The solutions for both half-space and thin film geometries are discussed in appendix B. We obtain,

$$
\frac{1}{\lambda_{\|}(\vec{H})}=\frac{1}{\lambda_{\|}}\left(1-\frac{H}{H_{0}}\right), \quad \vec{H} \| \text { node }
$$


and similarly for fields directed along an antinode,

$$
\frac{1}{\lambda_{\|}(\vec{H})}=\frac{1}{\lambda_{\|}}\left(1-\frac{1}{\sqrt{2}} \frac{H}{H_{0}}\right), \quad \vec{H} \| \text { antinode, }
$$

where $\lambda_{\|}$is the zero-field penetration depth at $T=0$ and $H_{0}=3 c v_{0} / 2 e \lambda_{\|}$is the characteristic field scale. Using $v_{0}=\mu \Delta_{0} / v_{f}^{*}, 1 / \lambda_{\|}^{2}=4 \pi e^{2} N_{f} \bar{v}_{f}^{2} / c^{2}, \xi_{\|}=\bar{v}_{f} / 2 \pi T_{c}$ and $H_{c}^{2} / 8 \pi=\frac{1}{2} N_{f} \Delta^{2}$ gives $H_{0} \simeq \frac{3}{2} \mu\left(\bar{v}_{f} / v_{f}^{*}\right)^{3} H_{c} \sim \mathcal{O}\left(H_{c}\right)$, where $H_{c}$ is the thermodynamic critial field, $\bar{v}_{f}$ is the $r m s$ average of the Fermi velocity and $v_{f}^{*}$ is the Fermi velocity at the node. We estimate $H_{c}(0) \simeq 8.5 k G$ and $H_{0} \simeq 3.4 T$ if we neglect the anisotropy of the Fermi velocity. Fermi surface anistropy can change the characteristic field significantly. Using a next-nearest neighbor tight-binding model for the Fermi surface fit to the LDA result for $\mathrm{YBa}_{2} \mathrm{Cu}_{3} \mathrm{O}_{7-\delta}$, [57] we estimate the anisotropy to be $v_{f}^{*} / \bar{v}_{f} \simeq 1.1$, and gives $H_{0} \simeq 2.5 T$; however, the anisotropy of $\vec{v}_{f}$ is sensitive to the hole concentration near half filling. Assuming $H_{0}=2.5 T$ and $H_{c 1}(0) \sim 250 G$ appropriate for twinned single crystals of YBCO, [58] then over the field range, $0 \leq H \leq H_{c 1}$, the change in $\lambda_{\|}(\vec{H})$ is of order $\delta \lambda_{\|} \simeq \lambda_{\|} H_{c 1} / H_{0} \simeq(1,500 \AA)(250 G) /\left(2.5 \times 10^{4} G\right) \simeq 15 \AA$. The magnitude of $\delta \lambda_{\|}(\vec{H})$ also depends on the measurement technique. If $\delta \lambda_{\|}(\vec{H})$ is measured from the inductive response of a low-frequency a.c. field, $\vec{A}_{\omega}$, in the presence of a parallel d.c. field, $\vec{v}_{s}$, then from eq. (62) $\delta \vec{j}_{\omega}=-\frac{e^{2}}{c} \rho\left\{1-2\left|\vec{v}_{s}\right| / v_{0}\right\} \vec{A}_{\omega}$; and the change in the penetration depth with the static field is a factor of 2 larger than the d.c. result in eqs. (66) and (67).

Observation of the anisotropy and linear field dependence of $\delta \lambda_{\| \mid}(\vec{H})$ could provide strong support for a $d_{x^{2}-y^{2}}$ order parameter. Below we consider thermal and impurity effects which might mask or wash-out the characteristic anistropy and linear field dependence characteristic of pure material at $T=0$.

\section{Thermal excitations: cross-over to analytic behavior}

At finite temperatures thermally excited quasiparticles occupy the upper branch of the counter-moving band shown in Fig. 10, and for $T \ll \Delta_{0}$ these thermal quasiparticles are predominantly in the nodal regions. In the limit $v_{f} v_{s} \ll \pi T$ the thermal excitations dominate and the nonlinear corrections can be obtained from a Taylor expansion in $\left(v_{f} v_{s} / \pi T\right)$. In the opposite limit $\pi T \ll v_{f} v_{s} \ll \pi T_{c}$, the non-thermal jets that give rise to the non-analytic backflow current dominate. Thus, at finite temperature there is a cross-over from the non-analytic result with $\vec{j}_{q p} \sim e \rho \vec{v}_{s}\left|v_{f} v_{s} / \Delta_{0}\right|$ for $\pi T \ll v_{f} v_{s} \ll \pi T_{c}$ to $\vec{j}_{q p} \sim e \rho \vec{v}_{s}\left(v_{f} v_{s} / \pi T\right)^{2}$ for $0<v_{f} v_{s} \ll \pi T$ (These expansions are discussed in appendix A). Hence, with decreasing surface field, $H$, the effective penetration depth also crosses over from a linear field dependence, $\delta \lambda_{\|} \simeq \lambda_{\|}\left(H / H_{0}\right)$, for $0<H_{T}<H \ll H_{c}$, to a quadratic dependence, $\delta \lambda_{\|} \sim\left(H / H_{T}\right)^{2}$ for $0<H<H_{T}$, where the cross-over scale is the field at which the flow energy per excitation becomes comparable to the thermal energy, $v_{f} v_{s} \simeq v_{f} \frac{e}{c} H_{T} \lambda \simeq \pi T$, or $H_{T} \simeq\left(T / \Delta_{0}\right) H_{0}$. For $T / \Delta_{0}=0.001$ (i.e. $T \sim 0.2 \mathrm{~K}$ and $T_{c}=100 \mathrm{~K}$ ) the cross-over field is $H_{T} \simeq 10 G$ with $H_{0}=10 k G$. However, at $T=2 \mathrm{~K}$, the cross-over moves to $H_{T} \simeq 100 \mathrm{G}$, which is a substantial fraction of $H_{c 1} \sim 200 G$ for the cuprates. At temperatures above $T \simeq 2 \mathrm{~K}$ the linear field region is essentially washed out by the thermal backflow current. Thus, it is essential to work at $T \ll T_{c}\left(H_{c 1} / H_{0}\right)$ in order to minimize the current from the thermally excited quasiparticles. Note that the restrictions on the temperature are more severe for observing the linear field dependence, compared to the linear temperature dependence at $H=0$, because a clean interpretation of the Meissner penetration length requires fields below the vortex nucleation field. If vortex nucleation could otherwise be suppressed, then the field range could be extended to $H \sim H_{0}$ and a linear field dependence would be observable over a much larger field range, and correspondingly the restriction on the low-field cross-over would be much less severe. In any event we typically assume a field range up to $H_{c 1} \simeq 250 G$ in our calculations and estimates.

In Fig. 13 we show the effect of thermal excitations on the velocity dependence of the effective superfluid density $\rho_{s}\left(T, v_{s}\right) \equiv j_{s} / v_{s}$ for $\vec{v}_{s}$ directed along a node. [16] The intercept shows the usual thermal reduction in $\rho_{s}$ at $v_{s}=0$. The cross-over from the linear field dependence at $v_{f} v_{s} \gg \pi T$ is clearly seen, and the arrows indicate the value of the cross-over velocity, $v_{T}=\pi T / v_{f}$. Figure 14 for the field-dependent Meissner penetration depth contains similar information. The $T=0$ result is our analytic solution to the nonlinear London equation; for the curves at $T \neq 0$ we converted the current-velocity relations with the same field scaling as we derived for the $T=0$ scale, $v_{s} / v_{0} \rightarrow H / H_{0}$. Stojković and Valls have recently solved the nonlinear London equations numerically at $T \neq 0$. [59] Our scaling assumption for $T \neq 0$ agrees well with their numerical solutions for $H_{T} \leq H_{c 1}$. Figure 14 gives the estimates of $\delta \lambda_{\|}$ for $H \simeq H_{c 1}$ for several temperatures. The two estimates at $T=0$ correspond to $H_{c 1} / H_{0}($ d.c. $)=0.01$, appropriate for the d.c. measurement of $\delta \lambda_{\|}$, and $H_{c 1} / H_{0}$ (a.c. $)=0.02$, appropriate for the a.c. measurement of $\delta \lambda_{\|}$. Thus, at $T \rightarrow 0$ we estimate a change in $\lambda_{\|} \sim 30 \AA$ in an a.c. measurement for fields directed along the node. At $T=0.4 K$ the cross-over is observable in the calculation, but we expect to see a clear linear field dependence over the field range 
up to $H_{c 1}$. At $T \simeq 1 K$ the resolution is reduced, $\delta \lambda_{\|} \simeq 20 \AA$ and a linear field dependence is observed over roughly $60 \%$ of the field range. However, by $T \simeq 4 \mathrm{~K}$ the cross-over field is $H_{T} \simeq H_{c 1}$; the linear field dependence is washed out and the change in $\lambda_{\|}$is less than $15 \AA$. These results are all reduced by a factor of $\simeq 1 / \sqrt{2}$ for a field along an antinode.

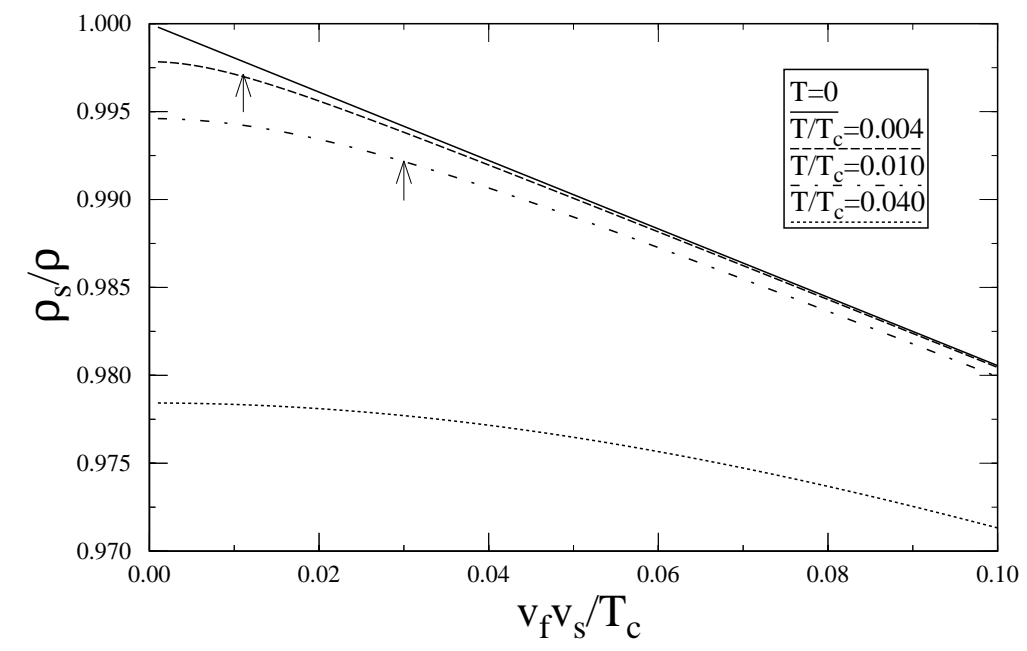

Fig. 13 Velocity and temperature dependence of the effective superfluid density, $\rho_{s}=j_{s} / v_{s}$, for $\vec{v}_{s} \|$ node in the clean limit. The cross-over velocities are indicated by the arrows.

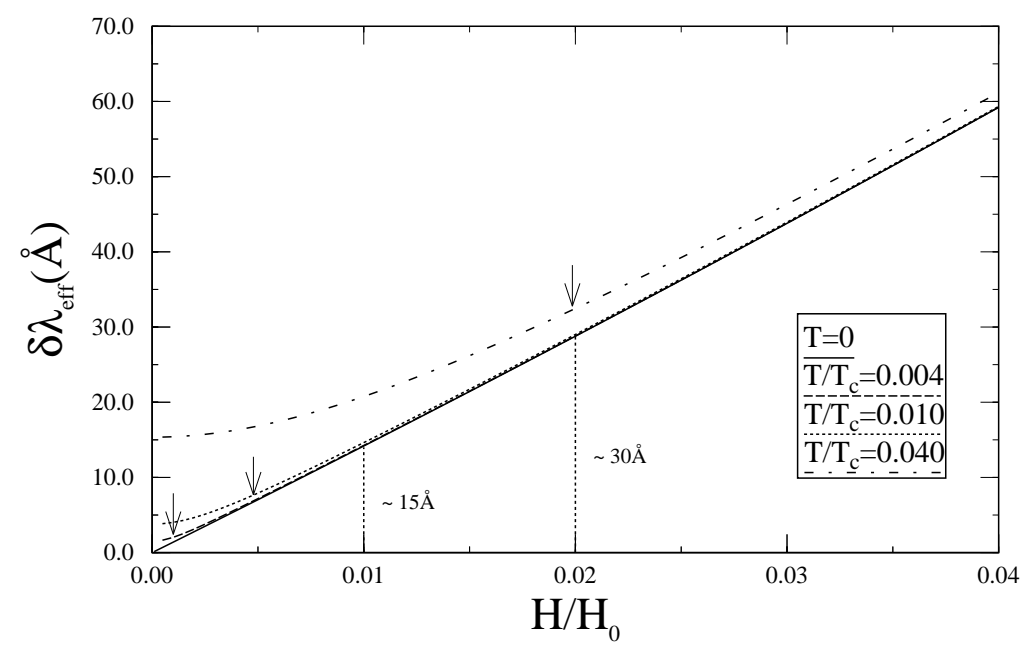

Fig. 14 Field dependence of the penetration depth for $\vec{H} \|$ node. The cross-over field, $H_{T}=\left(T / \Delta_{0}\right) H_{0}$, is indicated by an arrow. The verticle marker at 0.01 (0.02) corresponds to $H_{c 1} / H_{0}$ for a d.c. (a.c.) measurement of the penetration depth.

\section{Impurity scattering}

Quasiparticle scattering by impurities also removes the non-analyitc dependence of the current on the condensate flow velocity at sufficiently low $v_{s}$. At $T=0$ impurity scattering gives rise to a cross-over velocity $v_{s}^{*}$, or field 
$H^{*}=H_{0}\left(v_{s}^{*} / v_{0}\right)$, that is determined by the energy scale, $\varepsilon^{*}$, in the density of states $\overline{\mathcal{N}}(E)$; above $\varepsilon^{*} \ll v_{f} v_{s} \ll \pi T_{c}$ the excitations that are strongly affected by impurity scattering at energies $E \lesssim \varepsilon^{*}$ are only a small fraction of the non-thermal backflow current, while at small flow velocities, $v_{f} v_{s} \lesssim \varepsilon^{*}$ the excitations contributing to the non-thermal backflow current are strongly modified by impurity scattering.

The cross-over field scale at $T=0$ due to impurity scattering can be obtained from the general expression in eq. (52) for the current; the cross-over velocity is given by $v_{f} v_{s}^{*}=\varepsilon^{*}$, where $\varepsilon^{*}$ is the cross-over energy from eq. (48). In the Born limit $\left(\delta_{0} \ll 1\right)$ the cross-over scale,

$$
H^{*}=H_{0} \frac{\varepsilon^{*}}{\mu \Delta_{0}} \simeq \frac{2}{\mu} H_{0} e^{-\frac{\mu}{2} \pi \tau \Delta_{0}}=\frac{2}{\mu} H_{0} e^{-\frac{\mu}{2}\left(l_{i m p} / \xi_{0}\right)} \quad \text { (Born), }
$$

is exponentially small for $l_{i m p} \gg \xi_{0}$. Thus, the linear field dependence and anisotropy of the penetration depth will be unaffected by impurity scattering in the weak scattering limit. The cross-over field may be much higher, even in the dilute limit, for strong scattering. In the unitarity limit $(\delta \rightarrow \pi / 2)$ the cross-over field scale is

$$
H^{*} \simeq H_{0} \sqrt{\frac{\pi}{2 \ln \left(\Delta_{0} / \Gamma_{u}\right)} \frac{\Gamma_{u}}{\mu \Delta_{0}}} \quad \text { (Unitarity). }
$$

For $\Gamma_{u} / \Delta_{0}=10^{-4}$, which is a good bound for $\Gamma_{u}$ obtained from the data of Ref. ( [1]), we obtain, $H^{*} \simeq 2.6 \times 10^{-3} H_{0} \simeq$ $26 G$. Thus, even in the unitarity limit there is a large field range, $25 G \sim H^{*}<H<H_{c 1} \sim 250 G$ in which the linear field dependence of $\delta \lambda_{\|}(\vec{H})$ is expected to hold. Of course it is important to be at low temperatures in order to avoid the thermal cross-over. Figure 15 shows our numerical results for the field-dependent penetration depth (d.c.) calculated for $T / T_{c}=0.004$ and unitarity scattering. The field range is approximately $0<H \lesssim H_{c 1} \simeq 250 G$. In the clean limit $\left(\Gamma_{u}=0\right)$ the curvature at very low fields is due to the thermal cross-over discussed earlier. As the impurity concentration increases the curvature sets in at higher fields; the arrows indicate the impurity cross-over fields calculated from eq. (69); note the calculated cross-over field accurately reflects the field dependence obtained from the numerical calculations. The results for $\Gamma_{u} / \Delta_{0} \simeq 10^{-4}$ suggest that a nonlinear Meissner effect with a linear field dependence over $\sim 80 \%$ of the field range from zero to $H_{c 1}$ should be observable in single crystals of comparable purity to those of Ref. ( [1).

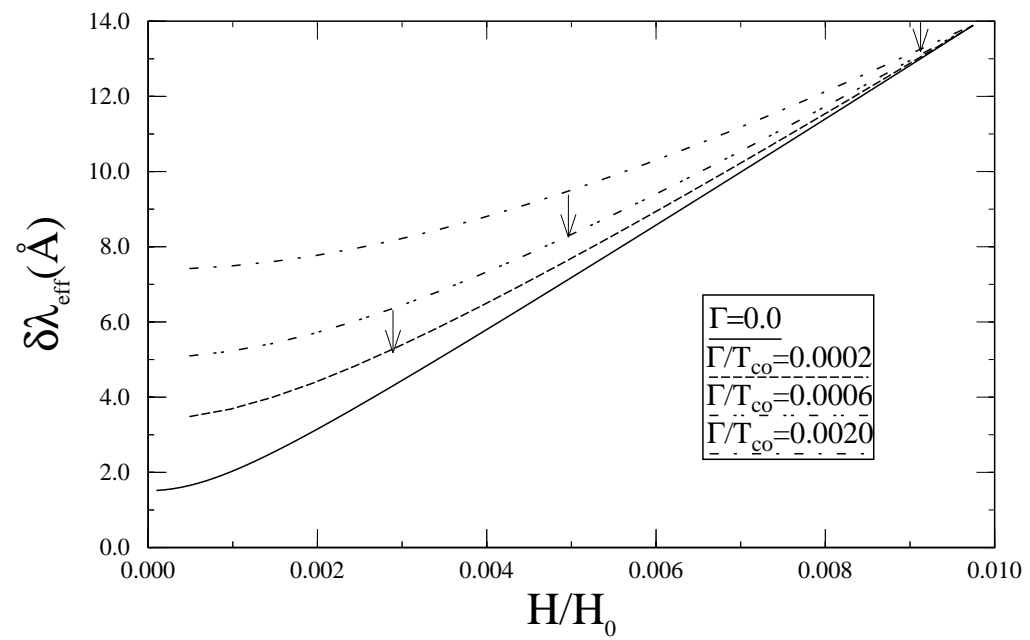

Fig. 15 Field-dependence of the penetration depth with unitarity scattering. The cross-over field $H^{*}$ is indicated by an arrow.

\section{ANISOTROPY OF THE IN-PLANE MAGNETIC TORQUE}

Another test of the presence of nodal lines associated with a $d_{x^{2}-u^{2}}$ order parameter would be to measure the magnetic anisotropy energy, or magnetic torque, for in-plane fields. 115. Consider a velocity field $\vec{v}_{s}=v_{x^{\prime}} \hat{x}^{\prime}+v_{y^{\prime}} \hat{y}^{\prime}$ 
that is not directed along a node or antinode. At $T=0$ the projections of the velocity along the nodal lines $\hat{x}^{\prime}$ and $\hat{y}^{\prime}$ give rise to two backflow jets of different magnitudes,

$$
\vec{j}_{q p}=-e N_{f} v_{f}^{2}\left[\left(\frac{v_{x^{\prime}}^{2}}{v_{o}}\right)\left(-\hat{x}^{\prime}\right)+\left(\frac{v_{y^{\prime}}^{2}}{v_{o}}\right)\left(-\hat{y}^{\prime}\right)\right] .
$$

The important feature is that the current is not parallel to the velocity field, except for the special directions along the nodes or antinodes. As a consequence the magnetic field in the screening layer, $\vec{b}$, is not parallel to the applied surface field, $\vec{H}$. This implies that there is an in-plane magnetic torque which acts to align the nodes of the gap, and therefore the crystal axes, with the surface field.

The magnetic free energy of the superconductor, in the presence of the surface field $\vec{H}$, is given by

$$
U=-\int_{V_{\text {film }}} d^{3} x \int_{0}^{H} \vec{M}\left(\vec{H}^{\prime}\right) \cdot d \vec{H}^{\prime} .
$$

The integration is carried out assuming that the orientation of $\vec{H}^{\prime}$ is fixed; $\vec{H}^{\prime}=H^{\prime}\left(\sin (\theta) \hat{x}^{\prime}-\cos (\theta) \hat{y}^{\prime}\right)$, and $\vec{M}(\vec{H})$ is the equilibrium magnetization for the given value of $\vec{H}$, which is easily be found by solving the nonlinear London equation in the film geometry (see appendix B). The resulting anisotropic contribution to the magnetic energy is obtained by integrating over the volume of the film, 4

$$
U_{a n}(\theta)=-\frac{H^{2}}{4 \pi} \frac{H}{H_{0}} A \lambda_{\|}\langle\Phi\rangle\left[\sin ^{3}(\theta)+\cos ^{3}(\theta)\right],
$$

where $A$ is the surface area of the film, and (see appendix B)

$$
\langle\Phi\rangle=2 \int_{0}^{d / 2 \lambda} d \zeta \Phi(\zeta)=\frac{8}{3}\left\{\frac{\sinh ^{4}\left(\frac{d}{4 \lambda}\right)\left[\frac{1}{2}+\cosh ^{2}\left(\frac{d}{4 \lambda}\right)\right]}{\cosh ^{3}\left(\frac{d}{2 \lambda}\right)}\right\} .
$$

In the thin and thick film limits we obtain,

$$
\langle\Phi\rangle= \begin{cases}\frac{1}{4}\left(\frac{d}{2 \lambda}\right)^{4} & , d \ll \lambda_{\|} \\ \frac{1}{3} & , d \gg \lambda_{\|} .\end{cases}
$$

Note that the anisotropy energy is minimized for field directions along the nodal lines, and is maximum for fields along the antinodes.

At finite temperature an analytic expression corresponding to eq. 70 ) is not available except in special limits ( see appendix A). In order to calculate the magnetic torque at finite temperature, we note that for $\left|v_{f} v_{s}\right| \ll \Delta_{0}$, we can write $j_{s x, y}=\rho_{s x, y} v_{s x, y}$ (we drop the primes on $x$ and $y$, but emphasize that the axes refer to two orthogonal nodal directions) with

$$
\frac{\rho_{s x, y}}{\rho}=1-g\left(v_{f} v_{s, y} / \mu \Delta_{0}\right),
$$

where $g(x)$ is a dimensionless function (see the subsections on 'thermal excitations' and 'impurity scattering' in section IV). At $T=0 g(x)=|x|$, manifesting the non-analytic behavior of the current, while at finite temperature $g(x)$ has a linear region for large $x\left(v_{f} v_{s} / T \gg 1\right)$, and crosses over to a quadratic region for small $x\left(v_{f} v_{s} / T \ll 1\right)$.

The flow velocity and field distribution are determined by the nonlinear London equation,

$$
\frac{\partial^{2} v_{s x, y}}{\partial z^{2}}-\frac{1}{\lambda_{\|}^{2}} v_{s, y}\left\{1-g\left(v_{f} v_{s} x, y / \mu \Delta_{0}\right)\right\}=0,
$$

where $\lambda_{\|}$is the penetration length in the limit of zero field. The solution for the velocity field can be obtained by performing a perturbation expansion in $v_{f} v_{s} / \mu \Delta_{0}$. For a superconductor occupying the half space $z>0$, the solution is

\footnotetext{
${ }^{4}$ The authors of Ref. ( 15 ) erred in calculating the magnetic anisotropy energy by a factor of 3 . The corrected result is given by eq. (72).
} 


$$
u_{x, y}(z)=\frac{v_{f} v_{s x, y}}{\mu \Delta_{0}}=u_{x, y 0} e^{-z / \lambda}+e^{-z / \lambda} \int_{0}^{z} \frac{d z^{\prime}}{\lambda} \frac{\mathcal{G}\left(u_{x, y 0} e^{-z^{\prime} / \lambda}\right)}{u_{x, y 0}} e^{2 z^{\prime} / \lambda},
$$

where $\mathcal{G}(x)=\int_{o}^{x} x^{\prime} g\left(x^{\prime}\right) d x^{\prime}$. The value of the $\vec{u}_{0}$ is determined by the boundary condition, $d v_{s, y} /\left.d z\right|_{z=0}= \pm \frac{e}{c} H_{y, x}$; thus,

$$
u_{x, y 0}=\mp \frac{H_{y, x}}{\frac{2}{3} H_{0}}+\frac{\mathcal{G}\left(\frac{H_{y, x}}{\frac{2}{3} H_{0}}\right)}{\frac{H_{y, x}}{\frac{2}{3} H_{0}}} .
$$

The magnetic torque can be obtained from the magnetic anisotropy energy, $\tau_{z}=-\partial U_{a n} / \partial \theta$, or equivalently $\vec{M} \times \vec{H}$. The integral of $\vec{b}=\vec{\nabla} \times \vec{A}$ can be expressed in terms of $\vec{v}_{s}$ at the surface, since $\int_{0}^{\infty} b_{x, y} d z= \pm \frac{c}{e} v_{s} y,\left.x\right|_{z=0}$. Thus, for a thick film

$$
\tau_{z}=2 \frac{1}{4 \pi}\left(\frac{2}{3} H_{0}\right)^{2} \lambda A\left[\frac{H_{y}}{H_{x}} \mathcal{G}\left(\frac{H_{x}}{\frac{2}{3} H_{0}}\right)+\frac{H_{x}}{H_{y}} \mathcal{G}\left(\frac{H_{y}}{\frac{2}{3} H_{0}}\right)\right],
$$

the torque can be calculated from a simple integration of the current-velocity relation. At $T=0$ the torque equation (79) reduces to

$$
\tau_{z}=\frac{1}{4 \pi} \lambda \frac{H}{H_{0}} H^{2} A \sin \theta \cos \theta(\sin \theta-\cos \theta) \quad, \quad 0 \leq \theta \leq \pi / 2,
$$

in agreement with the derivative of the anisotropy energy in eq. (72).

For $H=400 G, A=(2,000 \mu \mathrm{m})^{2}$ and $\lambda=1,400 \AA$, the zero temperature maximum magnetic torque $\tau_{z} \simeq$ $(1 / 12 \sqrt{3} \pi) H^{2}\left(H / H_{0}\right) A \lambda \sim 10^{-5}$ dyne $-\mathrm{cm} / \mathrm{rad}$. In Fig. 16 we show results for the magnitude of the torque at finite temperature. The torque is only weakly reduced for $T \lesssim 1 K$, but drops rapidly above $T \sim 1 K$.

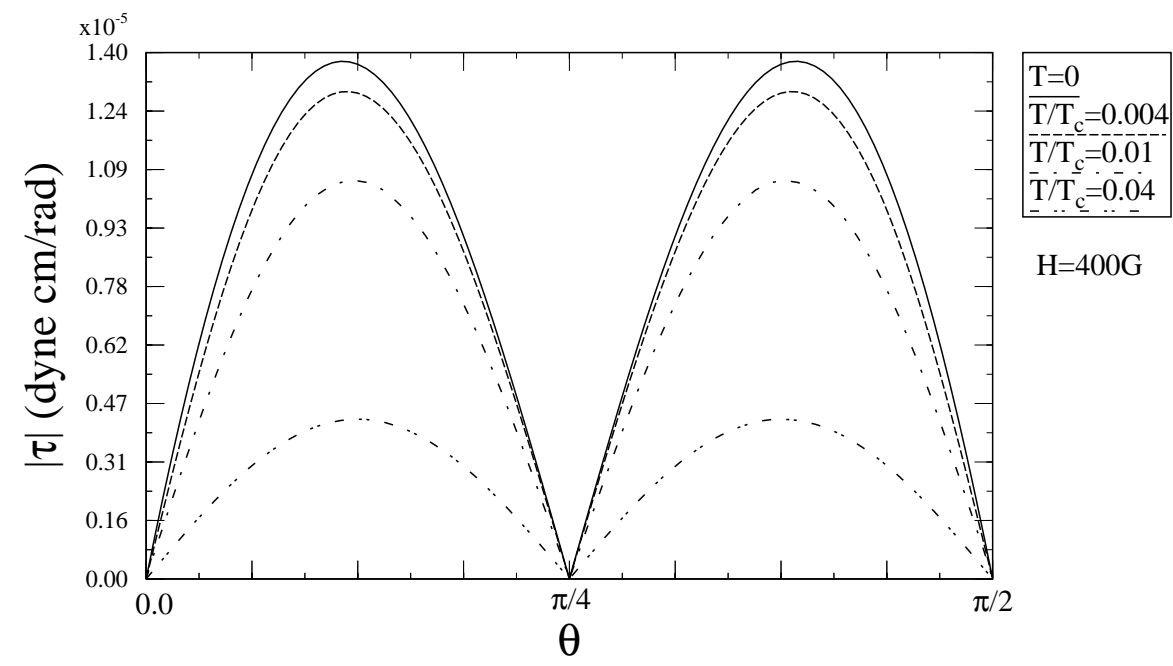

Fig. 16 Magnitude of torque as a function of $\theta$. Note that $\theta=0, \pi / 2$ correspond to node positions, $\theta=\pi / 4$ corresponds to an antinode position, and the maximum torque (for $T=0$ ) occurs at $\theta=\frac{1}{2} \sin ^{-1}(2 / 3) \simeq 21^{\circ}$. The torque has four fold symmetry and points to the nodal positions.

In order to maximize the torque it is desirable to suppress vortex nucleation so that the torque measurement can be performed at a higher magnetic field. In this respect, thin films with dimensions $d \leq \lambda$ might be desirable, because the vortex nucleation field is increased by roughly $(\lambda / d)$ in a thin film. The optimum geometry might be a superlattice of superconducting/normal layers with an S-layer thickness, $\xi \ll d<\lambda$. In this case the field at each SN interface is essentially the external field, and the anisotropy energy is enhanced by the number of S-layers. 
In a recent paper Buan, et al. reported [17] measurements of the transverse magnetic moment induced by an in-plane surface field in an untwinned single-crystal of $\mathrm{LuBa}_{2} \mathrm{Cu}_{3} \mathrm{O}_{7-\delta}$. The surface field was rotated in the a-b plane and the Fourier component of $M_{\perp}(\theta) \propto \cos (4 \theta)$ was extracted and compared with predictions based on the theory of Ref. ( 15]). Buan, et al. report a measured transverse magnetization signal $(\sim \cos (4 \theta))$ of $M_{\perp}^{\exp } \simeq 0.8 \times 10^{-6}$ emu at $H=300 \mathrm{G}$ and $T=2 \mathrm{~K}$, and a resolution limit of $0.3 \times 10^{-6} \mathrm{emu}$. Buan, et al. also report a theoretical value of $M_{\perp}^{\text {theory }} \simeq 2 \times 10^{-6} \mathrm{emu}$, for the same temperature and field, obtained from numerical solution of the London equation with the nonlinear current-velocity equation from Ref. ( [15]). This estimate is 2.5 times the experimental signal and nearly an order of magnitude above the resolution limit. The authors $\operatorname{conclude}$ that the $\cos (4 \theta) \operatorname{signal}$ is too small to be consistent with a pure d-wave pairing state, that there are no nodes in the gap and that the $\cos (4 \theta)$ signal is consistent with a higher harmonic of the $\cos (\theta)$ and $\cos (2 \theta)$ signals associated with the shape anisotropy of the crystal.

While it may be that the observed $\cos (4 \theta)$ signal is associated with extrinsic effects of geometry, the conclusion that the measurement rules out a pure d-wave state with nodes relies principally on $M_{\perp}^{\text {theory }} \gg M_{\perp}^{\text {exp }}$. The estimate of $M_{\perp}^{\text {theory }} \simeq 2 \times 10^{-6}$ emu at $T=2 K$ and $H=300 G$ is based on the following parameters: $\lambda_{c}=1 \mu m, \lambda_{\|}=1,700 \AA$ and geometric parameters for the crystal, $a=1.2 \mathrm{~mm}, b=0.9 \mathrm{~mm}$ and $c=0.07 \mathrm{~mm}$. The transverse magnetic moment per unit area is given by $M_{\perp}=\tau_{z} /($ Area $H)$, which is proportional to $H^{2} \lambda_{\|}^{2}$ from eq. (80) for $T \rightarrow 0$. Buan, et al. argue that finite size effects require that $\lambda_{\|}$in the formula for $M_{\perp}$ be replaced by an effective penetration depth $\lambda \simeq 4,000 \AA$ in order to account for c-axis currents. This procedure leads to an increase in the in-plane transverse magnetization due to current flow along the c-axis, which is opposite to what is expected. In particular, in a geometry with an aspect ratio, $c / a \simeq 0.07$, and a field lying in the ab-plane, say along the $b$-axis, the current flows predominantly along the $a$-axis. The 'return current' at the edges flows mainly along the c-axis. The main effect of the c-axis currents is a reduction in the area with current flow in the a-b plane, $A_{\text {eff }} \simeq A\left(1-2 \lambda_{c} / a\right)$. Thus, the in-plane, transverse magnetization for the semi-infinite geometry will correspondingly be reduced by roughly $A_{\text {eff }} / A=\left(1-2 \lambda_{c} / a\right)$. The reduction factor is tiny for the geometry of Ref. ( [17]), $A_{e f f} / A \simeq 0.999$; however, the theoretical value for $M_{\perp}^{\text {theory }}$ reported in Ref. ( 17]) is overestimated by a factor of $\left(\lambda / \lambda_{\|}\right)^{2} \simeq 5.5$. Dividing the theoretical estimate of $2 \times 10^{-\overline{6}}$ emu by 5.5 gives $M_{\perp}^{\text {theory }} \simeq 0.36 \times 10^{-6} \mathrm{emu}$, very near the resolution limit and below the observed signal at $H=300 \mathrm{G}$.

Thus, in our opinion this null result is inconclusive and does not force one to eliminate a pure d-wave state as a possible candidate for the order parameter of the cuprates. Experiments designed to minimize shape anisotropies at temperatures well below $T \simeq 2 K$ should be able to detect the intrinsic anisotropy associated with nodal excitations, should they exist.

\section{ACKNOWLEDGEMENTS}

We wish to thank Walter Hardy, Doug Bonn and their colleagues for sending us their results prior to publication. After completing this work, but before this manuscript was complete, we received a preprint from Stojković and Valls reporting numerical calculations of the field dependent penetration depth and transverse magnetization at finite temperatures. We thank the authors and note that our results are in good agreement when allowances are made for different choices of parameters (SV generally examined a much larger range of fields) and different details for the model of the gap. This work was supported by the National Science Foundation (DMR 91-20000) through the Science and Technology Center for Superconductivity. 


\section{APPENDIX A - TEMPERATURE AND FIELD DEPENDENCE OF THE CURRENT FOR A SUPERCONDUCTOR WITH LINE NODES}

In general we need to solve two problems, (i) the self-consistent calculation of the order parameter, $\Delta\left(\vec{p}_{f}\right)$, in the presence of a condensate flow field at finite temperature, and (ii) the computation of the temperature and field dependence of the current, $\vec{j}_{s}$, for a given $\Delta\left(\vec{p}_{f} ; \vec{v}_{s}, T\right)$. These problems are in general coupled because the flow field leads to suppression of the gap parameter, $\Delta$, which contributes to the field-dependence of the current. We show that at low temperatures, $T / T_{c} \ll 1$, and low fields, $v_{f} v_{s} / T_{c} \ll 1$, the contributions to the current from the gap suppression can be neglected.

We also discuss the functional form of the current at low temperatures and low velocity in the clean limit. In the limit $T / T_{c} \ll 1$ and $v_{f} v_{s} / T \ll 1$, an expansion in $v_{f} v_{s}$ is valid, and as a result, the leading field dependence of the superfluid density, $\rho_{s}$, is quadratic in the flow field; while in the limit $T / T_{c} \ll 1$ and $v_{f} v_{s} / T \gg 1$, the quasiparticle contribution to the current is non-analytic. For simplicity we confine ourselves to cases where $\vec{v}_{s}$ is parallel to a node or an antinode, in which case $\vec{j}_{s}$ and $\vec{v}_{s}$ are parallel so we drop the vector symbols.

In the limit $\left|v_{f} v_{s}\right| \ll T \ll \Delta_{0}$ we can expand $j_{s}$ in a power series in $v_{s} v_{f}$,

$$
j_{s}=-e N_{f} v_{f}^{2} v_{s}\left[1-\gamma_{1}\left(\frac{T}{\Delta_{0}}\right)-\gamma_{2} \frac{\left(v_{f} v_{s}\right)^{2}}{T \Delta_{0}}-\ldots\right]
$$

where $\Delta_{0}=\Delta_{0}\left(v_{s}, T\right)$, and $\gamma_{1}, \gamma_{2}$ are numerical coefficients of order one whose values depends on the angular slope parameter $\mu$ defined in eq.(45). The $\gamma_{1}$ term is the usual linear dependence of $\rho_{s}$ on $T$. The $\gamma_{2}$ term, being proportional to $1 / T$, signals the breakdown of the Taylor expansion in $v_{s}$ for sufficiently low temperatures.

The magnitude of the gap has the expansion,

$$
\Delta_{0}\left(v_{s}, T\right)=\Delta_{0}(0,0)\left[1-\alpha_{1}\left(\frac{T}{\Delta_{0}(0,0)}\right)^{3}-\alpha_{2}\left(\frac{v_{f} v_{s}}{\Delta_{0}(0,0)}\right)^{2}\left(\frac{T}{\Delta_{0}(0,0)}\right)-\ldots\right] .
$$

Thus, it is clear that the leading correction to $j_{s}$ from the flow field $v_{s}$ is given entirely by the $\gamma_{2}$ term; gap suppression is a higher-order correction.

In the limit $T \ll\left|v_{f} v_{s}\right| \ll \Delta_{0}$, we obtain,

$$
j_{s}=-e N_{f} v_{f}^{2} v_{s}\left[1-\gamma_{1}^{\prime} \frac{\left|v_{f} v_{s}\right|}{\Delta_{0}}-\gamma_{2}^{\prime}\left(\frac{T^{2}}{\left|v_{f} v_{s}\right| \Delta_{0}}\right)-\ldots\right] .
$$

The $\gamma_{1}^{\prime}$ term was calculated earlier, and the $\gamma_{2}^{\prime}$ term can be obtained from the Sommerfeld expansion of integrals involving the Fermi function, with $\left|v_{f} v_{s}\right|$ playing the role of the chemical potential for the quasiparticles. Note that both coefficents depend on the direction of $\vec{v}_{s}$. Also not that the $\gamma_{2}^{\prime}$ term gives a non-analytic current independent of $\left|v_{s}\right|$, but the result is only valid for $T \ll\left|v_{f} v_{s}\right|$. The gap in the above formula is

$$
\Delta_{0}\left(v_{s}, T\right)=\Delta_{0}(0,0)\left[1-\alpha_{1}^{\prime}\left(\frac{\left|v_{f} v_{s}\right|}{\Delta_{0}(0,0)}\right)^{3}-\alpha_{2}^{\prime}\left(\frac{\left|v_{f} v_{s}\right|}{\Delta_{0}(0,0)}\right)\left(\frac{T}{\Delta_{0}(0,0)}\right)^{2}-\ldots\right],
$$

which gives higher-order corrections to $j_{s}$. The quadratic correction to $j_{s}$ is unaffected by the gap suppression; the lowest-order temperature correction is given by the term $\propto \gamma_{2}^{\prime}\left(\frac{T^{2}}{\left|v_{f} v_{s}\right| \Delta_{0}}\right)$.

\section{APPENDIX B - NONLINEAR LONDON EQUATIONS}

\section{Conventional superconductor with an isotropic gap}

We include solutions of the nonlinear London equations for a parallel surface magnetic field penetrating into a superconductor. Starting from Ampere's equation

$$
\vec{\nabla} \times(\vec{\nabla} \times \vec{A})=\frac{4 \pi}{c} \vec{j}_{s}
$$

consider first an isotropic superconductor with a conventional gap. The constitutive equation for the current is given by eq.(58). In the absence of phase vortices, $\vec{\nabla} \times \vec{\nabla} \chi=0$, we obtain the nonlinear London equation, 


$$
\nabla^{2} \vec{v}_{s}-\frac{1}{\lambda^{2}} \vec{v}_{s}\left\{1-\alpha(T)\left(\frac{v_{s}}{v_{c}}\right)^{2}\right\}=0,
$$

where we have chosen the gauge, $\vec{\nabla} \cdot \vec{v}_{s}=0$. This gauge choice is consistent with current conservation provided that the current is transverse to the spatial variation of the field, which requires that there be no vortices present. Consider the geometry in which the superconductor occupies the half-space $z>0$. A surface field $\vec{H}=H \hat{x}$ is parallel to the interface, and the screening current $\vec{v}_{s}$ is then parallel to $\hat{y}$. The field in the superconductor is given by $\vec{b}=\frac{c}{e}\left(d v_{s} / d z\right) \hat{x}$. Continuity of the parallel field at the interface imposes the boundary condition

$$
\left.\frac{d v_{s}}{d z}\right|_{z=0}=\frac{e}{c} H .
$$

The field, and therefore the screening current, also vanish deep inside the superconductor. We introduce a dimensionless velocity, $u=\sqrt{\alpha} v_{s} / v_{c}$, and distance, $\zeta=z / \lambda(T)$; the differential equation for $u(\zeta)$ becomes,

$$
\frac{d^{2} u}{d \zeta^{2}}-u\left(1-u^{2}\right)=0
$$

with the boundary conditions,

$$
\left.\frac{d u}{d \zeta}\right|_{\zeta=0}=\frac{e}{c} \frac{\sqrt{\alpha} v_{f} H \lambda}{\Delta} \equiv h_{0} \quad, \quad u(\zeta \rightarrow \infty)=0 .
$$

A first integral is obtained by multiplying by $d u / d \zeta$, integrating, and applying the asymptotic boundary condition, $u \rightarrow 0$ as $z \rightarrow \infty$, to obtain,

$$
\frac{d u}{d \zeta}=-u \sqrt{1-\frac{1}{2} u^{2}}
$$

In the physically relevant limit, $|u| \ll 1$, we replace $\left(1-\frac{1}{2} u^{2}\right)^{1 / 2} \rightarrow\left(1-\frac{1}{4} u^{2}\right)$, and integrate to obtain,

$$
u=\frac{u_{0}}{\sqrt{u_{0}^{2} / 4+\left(1-u_{0}^{2} / 4\right) e^{2 \zeta}}} .
$$

The dimensionless magnetic field is given by

$$
h(\zeta)=\frac{d u}{d \zeta}=-\frac{u_{0}\left(1-u_{0}^{2} / 4\right) e^{2 \zeta}}{\left[u_{0}^{2} / 4+\left(1-u_{0}^{2} / 4\right) e^{2 \zeta}\right]^{3 / 2}},
$$

and the constant $u_{0}$ is determined by the boundary condition on magnetic field at the interface, eq.(89); $h_{0}=$ $-u_{0}\left(1-u_{0}^{2} / 4\right)$, or for weak nonlinearity, $u_{0} \simeq-h_{0}\left(1+h_{0}^{2} / 4\right)$, which yields the solution,

$$
h=\frac{h_{0} e^{2 \zeta}}{\left[h_{0}^{2} / 4+\left(1-h_{0}^{2} / 4\right) e^{2 \zeta}\right]^{3 / 2}} .
$$

Note that if we neglect the $h_{0}^{2}$ terms in the denominator we recover the linear response solution to the London equation, $h=h_{0} e^{-\zeta}$.

A strong surface field produces a reduction in the screening current at the surface and a correspondingly longer initial penetration depth. We take the initial decay rate to define the effective penetration depth,

$$
\frac{1}{\lambda_{e f f}}\left(h_{0}\right) \equiv \frac{1}{\lambda}\left[-\frac{1}{h_{0}} \frac{d h}{d \zeta}\right]=\frac{1}{\lambda}\left[1-\frac{3}{4} h_{0}^{2}\right] .
$$

This definition is equivalent to identifying the effective penetration depth with the surface impedance, i.e. $1 / \lambda_{\text {eff }}\left(h_{0}\right) \propto j_{s}(0) / H$. Note that the effective penetration depth increases with field as expected. In physical units

$$
h_{0}=\lambda \sqrt{\alpha} \frac{v_{f}}{\Delta} \frac{e}{c} H=\sqrt{\alpha} \frac{H}{H_{0}},
$$

where $H_{0}=c \Delta / e \lambda v_{f} \simeq \phi_{0} / \lambda \xi \simeq H_{c}$ and $H_{c}$ is the thermodynamic critial field. 


\section{$d_{x^{2}-y^{2}}$ superconductors}

Now consider a superconductor with a $d_{x^{2}-y^{2}}$ order parameter at $T=0$. Choose a coordinate system in which the nodes of the gap are directed along the $\pm \hat{x}$ and $\pm \hat{y}$ axes. The nonlinear London equations for the corresponding projections of the condensate velocity are

$$
\frac{d^{2} u_{i}}{d \zeta^{2}}-u_{i}\left\{1-u_{i}\right\}=0 \quad i=x, y
$$

where $u_{i}=v_{i} / v_{0}, \zeta=z / \lambda_{\|}$, and the velocity and length scales are $v_{0}=\mu \Delta_{0} / v_{f}$ and $\lambda=\lambda_{\|}$. These equations are to be solved subject to the boundary condition $\vec{H}=\vec{\nabla} \times\left.\vec{A}\right|_{z=0}$, which becomes,

$$
\left.\frac{d u_{x}}{d \zeta}\right|_{\zeta=0}=-h_{0} \cos \theta \quad,\left.\quad \frac{d u_{y}}{d \zeta}\right|_{\zeta=0}=-h_{0} \sin \theta
$$

where $\theta$ is the angle of $\vec{H}$ measured relative to the node along $-\hat{y}$, and $h_{0}=\frac{e}{c} \lambda H / v_{0}$. Note that $h_{0} \sim H / H_{0}$. The differential equation can be solved perturbatively; the first integral is

$$
\frac{d u_{i}}{d \zeta}=-u_{i}\left(1-\frac{2}{3} u_{i}\right)^{1 / 2}
$$

which can be integrated to give

$$
u_{i}(\zeta)=\frac{u_{i 0}}{\left.\frac{1}{3} u_{i 0}+\left[\left(1-\frac{1}{3} u_{i 0}\right) \cosh (\zeta)+\sqrt{1-\frac{2}{3} u_{i 0}} \sinh (\zeta)\right)\right]},
$$

where $u_{i 0}$ is the value of the velocity field at $\zeta=0$, which is fixed by the boundary conditions on the field. In the limit $h_{0} \ll 1$ we obtain

$$
\begin{aligned}
& u_{x 0}=h_{0} \cos \theta\left(1+\frac{1}{3} h_{0} \cos \theta\right), \\
& u_{y 0}=h_{0} \sin \theta\left(1+\frac{1}{3} h_{0} \sin \theta\right) .
\end{aligned}
$$

The magnetic field in the screening layer, $\vec{b}=\vec{\nabla} \times \vec{A}$, is given by

$$
\begin{aligned}
& b_{x} / H=-\frac{1}{h_{0}}\left(\frac{d u_{y}}{d \zeta}\right) \simeq+\sin \theta e^{-\zeta}\left(1+\frac{2}{3} h_{0} \sin \theta\left[1-e^{-\zeta}\right]\right), \\
& b_{y} / H=+\frac{1}{h_{0}}\left(\frac{d u_{x}}{d \zeta}\right) \simeq-\cos \theta e^{-\zeta}\left(1+\frac{2}{3} h_{0} \cos \theta\left[1-e^{-\zeta}\right]\right) .
\end{aligned}
$$

Note that the field in the screening layer is not parallel to the applied surface field. This leads to an in-plane magnetic torque acting on the superconductor which tends to align the nodes of the order parameter and the surface field.

Field penetration in a thin film $d_{x^{2}-y^{2}}$ superconductor

A geometry in which the torque anisotropy may be measured is a film of thickness $d \sim \lambda_{\|}$. The field $\vec{H}$ is oriented as in the half-space geometry, parallel to the $C u O$ planes and the surfaces of the film. Choose the origin at the center of the film; the boundary conditions for both interfaces are now,

$$
\left.\frac{d u_{x}}{d \zeta}\right|_{ \pm d / 2 \lambda}=-h_{0} \cos \theta \quad,\left.\quad \frac{d u_{y}}{d \zeta}\right|_{ \pm d / 2 \lambda}=-h_{0} \sin \theta .
$$


We solve the differential equation perturbatively. Let $u_{i}(\zeta)=L_{i}(\zeta)+\alpha_{i}(\zeta)$, where $L_{i}(\zeta)$ is the solution to the boundary-value problem for the linearized differential equations, i.e.

$$
L_{i}(\zeta)=a_{i} \sinh (\zeta)
$$

with $a_{i}$ fixed by the boundary conditions,

$$
a_{x} \cosh \left(\frac{d}{2 \lambda}\right)=-h_{0} \cos \theta \quad, \quad a_{y} \cosh \left(\frac{d}{2 \lambda}\right)=-h_{0} \sin \theta .
$$

The perturbation satisfies the inhomogeneous equation,

$$
\frac{d^{2} \alpha_{i}}{d \zeta^{2}}-\alpha_{i}=-L_{i}^{2} \quad i=x, y
$$

The solutions are obtained by writing $\alpha(\zeta)=\mathcal{H}(\zeta) \phi(\zeta)$, where $\mathcal{H}(\zeta)$ is a solution to the homogeneous equation. The function $\phi(\zeta)$ then satisfies,

$$
\phi^{\prime \prime}+\frac{2 \mathcal{H}^{\prime}}{\mathcal{H}} \phi^{\prime}=-L^{2} / \mathcal{H}
$$

The first-order equation for $\phi^{\prime}$ is obtained by multiplying eq. (108) by the integrating factor $\mathcal{H}^{2}$,

$$
\phi^{\prime}(\zeta)=\frac{-1}{\mathcal{H}(\zeta)^{2}} \int_{0}^{\zeta} L^{2}(x) \mathcal{H}(x) d x .
$$

The boundary conditions are satisfied at both interfaces by choosing the homogeneous solution to be, $\mathcal{H}=\sinh (\zeta)$, in which case

$$
\phi^{\prime}(\zeta)=\frac{-a^{2}}{\mathcal{H}(\zeta)^{2}} \int_{0}^{\zeta} \sinh ^{3}(x) d x=-\frac{a^{2}}{3}\left[\cosh (\zeta)-\operatorname{sech}^{2}(\zeta / 2)\right] .
$$

One additional integration yields

$$
\phi(\zeta)-\phi_{0}=-\frac{a^{2}}{3}[\sinh (\zeta)-2 \tanh (\zeta / 2)],
$$

where $\phi_{0}$ is also chosen such that the full nonlinear solution satisfies the boundary conditions. The resulting solutions for the magnetic field are

$$
\begin{gathered}
b_{x} / H=-\frac{1}{h_{0}}\left(\frac{d u_{y}}{d \zeta}\right)=+\sin \theta\left[\beta(\zeta)+h_{0} \sin \theta \Phi(\zeta)\right] \\
b_{y} / H=+\frac{1}{h_{0}}\left(\frac{d u_{x}}{d \zeta}\right)=-\cos \theta\left[\beta(\zeta)+h_{0} \cos \theta \Phi(\zeta)\right] \\
\beta(\zeta)=\cosh (\zeta) / \cosh \left(\frac{d}{2 \lambda}\right), \\
\Phi(\zeta)=\frac{8}{3} \operatorname{sech}^{3}\left(\frac{d}{2 \lambda}\right) \times\left\{\cosh (\zeta) \sinh ^{3}\left(\frac{d}{4 \lambda}\right) \cosh \left(\frac{d}{4 \lambda}\right)-\cosh \left(\frac{d}{2 \lambda}\right) \sinh ^{3}\left(\frac{\zeta}{2}\right) \cosh \left(\frac{\zeta}{2}\right)\right\}
\end{gathered}
$$

Note that the nonlinear correction is largest at a distance of order $\lambda / 2$ from the interface. 
[1] Hardy, W. N., Bonn, D. A., Morgan, D. C., Liang, R., and Zhang, K. Phys. Rev. Lett., 70, $3999,1993$.

[2] D. A. Wollman, D. J. VanHarlingen, Lee, W. C., Ginsberg, D. M., and Leggett, A. J. Phys. Rev. Lett., 71, $2134,1993$.

[3] Bickers, N. E., Scalapino, D. J., and Scalettar, R. T. Int. Journ. Mod. Phys., B1, 687, 1987.

[4] Moriya, T., Takahashi, Y., and Ueda, K. J. Phys. Soc. Japan, 59, 2905, 1990.

[5] Monthoux, P., Balatsky, A. V., and Pines, D. Phys. Rev. Lett., 67, 3448, 1991.

[6] Gor'kov, L. Sov. Sci. Rev. A., 9, 1, 1987.

[7] Sigrist, M. and Ueda, K. Rev. Mod. Phys., 63, 239, 1991.

[8] Sauls, J. Adv. Phys., 43, 113, 1994.

[9] Chaudhari, P. and Lin, S.-Y. Phys. Rev. Lett., 72, 1084, 1994.

[10] Tsuei, C. C., Kirtley, J. R., Chi, C. C., Yu-Jahnes, L. S., Gupta, A., Shaw, T., Sun, J. Z., and Ketchen, M. B. preprint, 1994.

[11] Bonn, D. A., Kamal, S., Zhang, K., Liang, R., Baar, D. J., Klein, E., and Hardy, W. N. preprint, 1994.

[12] Lee, J., Paget, K., and Lemberger, T. R. preprint, 1994.

[13] Sun, A. G., Gajewski, D. A., Maple, M. B., and Dynes, R. C. Phys. Rev. Lett., 72, 2267, 1994.

[14] Bonn, D. A., Morgan, D. C., Zhang, K., Liang, R., Baar, D. J., and Hardy, W. N. J. Phys. Chem. Solids, 54, $1297,1993$.

[15] Yip, S. K. and Sauls, J. A. Phys. Rev. Lett., 69, 2264, 1992.

[16] Xu, D., Yip, S. K., and Sauls, J. A. Physica B, 194-196, 1595, 1994.

[17] Buan, J., Stojkovic, B., Israeloff, N. E., Goldman, A. M., Valls, O., Liu, J., and Shelton, R. Phys. Rev. Lett., 72, 2632, 1994.

[18] Volovik, G. and Gor'kov, L. Sov. Phys. JETP Lett., 39, 674, 1984.

[19] Yip, S. and Garg, A. Phys. Rev., B48, 3304, 1993.

[20] Takigawa, M., Hammel, P. C., Heffner, R. H., and Fisk, Z. Phys. Rev., B39, 7371, 1989.

[21] Barrett, S. E., Durand, D. J., Pennington, C. H., Slichter, C. P., Friedmann, T. A., Rice, J. P., and Ginsberg, D. M. Phys. Rev., B41, 6283, 1990.

[22] Annett, J., Goldenfeld, N., and Renn, S. R., "The pairing state of $\mathrm{YBa}_{2} \mathrm{Cu}_{3} \mathrm{O}_{7-\delta}$," in Physical Properties of High Temperature Superconductors II (Ginsberg, D. M., ed.), 571, World Scientific, 1990.

[23] Serene, J. W. and Rainer, D. Phys. Rep., 101, 221, 1983.

[24] Rainer, D. in Progress in Low Temperature Physics, vol. 10, pp. 371-424, Elsevier Science Publishers B.V., 1986.

[25] Rainer, D. and Sauls, J. A. in Lecture Notes for the 1992 Spring School on Condensed Matter Physics, held in Trieste, Italy, Singapore: World Scientific Publishers, 1994.

[26] Eilenberger, G. Z. Physik, 214, 195, 1968.

[27] Gorkov, L. Sov.Phys. JETP, 36, 1364, 1959.

[28] Buchholtz, L. J. and Rainer, D. Z. Phys., B35, 151, 1979.

[29] Buchholtz, L. J. and Zwicknagl, G. Z. Phys., B23, 5788, 1981.

[30] Gor'kov, L. and Kalugin, P. A. Sov. Phys. JETP Lett., 41, 254, 1985.

[31] Anderson, P. W. J. Phys. Chem. Solids, 11, 26, 1959.

[32] Abrikosov, A. A. and Gorkov, L. P. Sov. Phys. JETP, 9, 220, 1959.

[33] Abrikosov, A. A. and Gorkov, L. P. Sov. Phys. JETP, 12, 1243, 1961.

[34] Leggett, A. J. Phys. Rev., 140, 1869, 1965.

[35] Gross, F., Chandrasekhar, B. S., Einzel, D., Hirschfeld, P. J., Andres, K., Ott, H. R., Fisk, Z., Smith, J., and Beuers, J. Z. Phys., B64, 175, 1986.

[36] Millis, A. J., Sachdev, S., and Varma, C. M. Phys. Rev., B37, 4975, 1988.

[37] Radtke, R. J., Levin, K., and Norman, H.-B. S. M. R. Phys. Rev. B, 48, 653, 1993.

[38] Preosti, G., Kim, H., and Muzikar, P. preprint, 1994.

[39] Fehrenbacher, R. and Norman, M. R. preprint, 1994.

[40] Choi, C. and Muzikar, P. Phys. Rev., B37, 5947, 1988.

[41] Prohammer, M. and Carbotte, J. P. Phys. Rev. B, 43, 5370, 1991.

[42] Hirschfeld, P. and Goldenfeld, N. Phys. Rev. B, 48, 4219, 1993.

[43] Sonier, J. E., Kiefl, R. F., Brewer, J. H., Bonn, D. A., Carolan, J. F., Chow, K. H., Dosanjh, P., Hardy, W., Liang, R., MacFarlane, A., Mendels, P., Morris, G. D., Riseman, T., and Schneider, J. W. Phys. Rev. Lett., 72, $744,1994$.

[44] Harshman, D., Scheemeyer, L., Waszcak, J., Aeppli, G., Cava, R., Batlogg, B., and Rupp, L. Phys. Rev. B, 39, 851, 1989.

[45] Chakravarty, S., Sudbø, A., and Anderson, P. W. Science, 261, 337, 1993.

[46] Laughlin, R. preprint, 1994.

[47] Fiory, A. T., Hebard, A. F., Mankiewich, P. M., and Howard, R. E. Phys. Rev. Lett., 61, 1419, 1988. 
[48] Annett, J., Goldenfeld, N., and Renn, S. R. Phys. Rev., B43, 2778, 1991.

[49] Pond, J. M., Caroll, K. R., and Horwitz, J. S. Appl. Phys. Lett., 59, 3033, 1991.

[50] Anlage, S. M. and Wu, D.-H. J. Supercon., 5, 395, 1992.

[51] Achir, D., Poirier, M., Bonn, D. A., Liang, R., and Hardy, W. Phys. Rev. B, 48, 13184, 1993.

[52] Lee, J. Y. and Lemberger, T. R. Appl. Phys. Lett, 62, 2419, 1993.

[53] Volovik, G. E. and Mineev, V. P. Sov. Phys. JETP, 54, 524, 1981.

[54] Combescot, R. and Dombre, T. Phys. Rev., 33, 79, 1986.

[55] Muzikar, P. and Rainer, D. Phys. Rev., B27, 4243, 1983.

[56] Choi, C. and Muzikar, P. Phys. Rev., B36, 54, 1987.

[57] Radtdke, R. J., Ullah, S., Levin, K., Schuttler, H., and Norman, M. Phys. Rev. B, 46, 11975, 1992.

[58] Umezawa, A., Crabtree, G. W., Welp, U., Kwok, W. K., and Vandervoort, K. G. Phys. Rev. B, 42 , $8744,1990$.

[59] Stojković, B. and Valls, O. preprint, 1994. 